International Journal of Bifurcation and Chaos, Vol. 15, No. 7 (2005) 2109-2129

(c) World Scientific Publishing Company

\title{
REALIZATION AND BIFURCATION OF BOOLEAN FUNCTIONS VIA CELLULAR NEURAL NETWORKS*
}

\author{
FANGYUE CHEN ${ }^{\dagger}$ \\ Department of Mathematics, Zhejiang Normal University, \\ Jinhua, Zhejiang, 321004, P. R. China \\ fychen@zjnu.cn \\ GUANRONG CHEN \\ Department of Electronic Engineering, \\ City University of Hong Kong, P. R. China \\ gchen@ee.cityu.edu.hk
}

Received May 4, 2004; Revised May 22, 2004

\begin{abstract}
In this work, we study the realization and bifurcation of Boolean functions of four variables via a Cellular Neural Network (CNN). We characterize the basic relations between the genes and the offsets of an uncoupled CNN as well as the basis of the binary input vectors set. Based on the analysis, we have rigorously proved that there are exactly 1882 linearly separable Boolean functions of four variables, and found an effective method for realizing all linearly separable Boolean functions via an uncoupled CNN. Consequently, any kind of linearly separable Boolean function can be implemented by an uncoupled CNN, and all CNN genes that are associated with these Boolean functions, called the CNN gene bank of four variables, can be easily determined. Through this work, we will show that the standard CNN invented by Chua and Yang in 1988 indeed is very essential not only in terms of engineering applications but also in the sense of fundamental mathematics.
\end{abstract}

Keywords: CNN; CNN gene; CNN gene bank; linearly separable Boolean function; bifurcation.

\section{Introduction}

Cellular Neural Networks (CNN) were originally introduced by Chua and Yang [1988a, 1988b] as an array of dynamical systems, called cells. In a twodimensional (2-D) configuration, it can be described by the following dynamical equations [Chua, 1997]:

$$
\begin{aligned}
\frac{d x_{i, j}}{d t}= & -x_{i, j}+z+\sum_{C_{k, l} \in S_{i, j}} a_{k, l} y_{i+k, j+l} \\
& +\sum_{C_{k, l} \in S_{i, j}} b_{k, l} u_{i+k, j+l}, \quad i, j \in Z^{2}
\end{aligned}
$$

with the output equations

$$
y_{i, j}=f\left(x_{i, j}\right)=\frac{1}{2}\left(\left|x_{i, j}+1\right|-\left|x_{i, j}-1\right|\right)
$$

where $S_{i, j}$ is the sphere of influence of radius $r=1$; $x_{i, j}, y_{i, j}, u_{i, j}$ and $z$ are scalars, called respectively state, output, input and threshold of cell $C_{i, j} ; a_{k, l}$ and $b_{k, l}$ are scalars synaptic weights.

A standard CNN is uniformly defined by a string of "19" real numbers, called a CNN gene, i.e. a uniform threshold $z$, nine feedback synaptic weights $a_{k, l}$, and nine control synaptic weights $b_{k, l}$, because the string completely determines the

\footnotetext{
*Work jointly supported by Hong Kong RGC (Grant CityU 1115/03E) and NSFC (Grant No. 10271121).

${ }^{\dagger}$ Author for correspondence.
} 
properties of the CNN. The universe of all CNN genes is called the CNN genome. Many real-world applications, from image processing to brain science to pattern recognition, can be easily implemented by a single CNN gene or a CNN "program" defined by a string of $\mathrm{CNN}$ genes called a CNN chromosome.

A Boolean function of $n$ variables is defined as the following binary map:

$$
F:\{-1,1\}^{n} \rightarrow\{-1,1\}, \quad F\left(u_{1}, u_{2}, \ldots, u_{n}\right)=v
$$

where $\left(u_{1}, u_{2}, \ldots, u_{n}\right) \in\{-1,1\}^{n}$ and $v \in\{-1,1\}$. Obviously, there exist $2^{2^{n}}$ Boolean functions for any given $n \in N$.

A CNN gene $G$ is said to be Boolean if, and only if, given any binary input image $U=\left\{u_{i, j} \in\right.$ $\{-1,1\}\}$, the steady-state output $y_{i, j}(\infty)$ of each cell $C_{i, j}$ is also binary, and can be uniquely determined by the input pattern of only those $C_{k, l}$ that are located inside the sphere of influence $S_{i, j}$ of $C_{i, j}$ [Chua, 1999].

It is known that only linearly separable CNN genes or linearly separable Boolean functions can be realized by an uncoupled standard CNN. In other words, the class of all uncoupled CNNs with binary inputs and outputs is identical to the linearly separable class of Boolean functions with respect to Boolean input-output maps [Chua, 1997; Chua et al., 2002; Julian et al., 2003]. It is also known that the linearly separable genes are very important for constructing the CNN chromosome; for example, the well-known game-of-life chromosome contains two linearly separable genes and a logical AND gene [Berlekamp et al., 1982; Chua, 1999].

Observe, on the other hand, that the number of Boolean functions quickly increases as the number of variables increases; for instance, there are $2^{2^{9}}=2^{512} \approx 1.34078 \times 10^{154}$ distinct Boolean functions or Boolean genes when $n=9$, which is a number tremendously greater than the size or age of the universe [Chua et al., 2002]. Therefore, a realization of the Boolean functions is a very important but also extremely difficult task.

How many distinct linearly separable CNN genes are there for $n$ input variables? That is, how many Boolean functions of $n$ variables can be realized by an uncoupled CNN? The known results are that there are 14 linearly separable CNN genes of two variables and there are 104 linearly separable ones of three variables [Chua, 1997, 1999]. The corresponding results on linearly separable CNN genes of four or more variables remain a question to be answered today.

In this paper, we study the realization problem for linearly separable Boolean functions of four variables via an uncoupled $\mathrm{CNN}$, and analyze the bifurcation of their genes. Because the CNN of four input variables agrees with the simplest 2-D network model, a realization of linearly separable Boolean genes is very essential. In this work, we not only rigorously prove that there are exactly 1882 linearly separable CNN genes in the family of $2^{2^{4}}=65536$ Boolean functions of four variables and their bifurcations, but also build up a complete CNN gene bank, which contains all the linearly separable genes of this kind.

The rest of this paper is organized as follows. Section 2 characterizes some essential properties of the input vector set and the structures of the uncoupled CNN of four input variables. Section 3 gives a main method of realization and bifurcation of these linearly separable Boolean functions. Section 4 lists one part of linearly separable Boolean genes of four variables and the binary decoding tapes as well as the decimal codes of the corresponding CNN output patterns. All these genes constitute a complete CNN gene bank of four variables, a part of the CNN genome. Finally, Sec. 5 presents some conclusions.

\section{Some Essential Properties of an Uncoupled CNN}

The standard uncoupled CNN is described by

$$
\begin{aligned}
\frac{d x_{i, j}}{d t}= & -x_{i, j}+z+a f\left(x_{i, j}\right) \\
& +\sum_{|k| \leq 1,|l| \leq 1} b_{k, l} u_{i+k, j+l}, \quad i, j \in Z^{2}
\end{aligned}
$$

namely, the feedback template and the control template in its gene \begin{tabular}{|l|l|l|l|l|l|}
\hline $\mathrm{z}$ & $\mathrm{B}$ & $\mathrm{A}$ & $\mathrm{A}$ & are \\
\hline
\end{tabular}

$$
\mathrm{A}=\left[\begin{array}{lll}
0 & 0 & 0 \\
0 & a & 0 \\
0 & 0 & 0
\end{array}\right]
$$

and

$$
\mathrm{B}=\left[\begin{array}{ccc}
b_{1,-1} & b_{1,0} & b_{1,1} \\
b_{0,-1} & b_{0,0} & b_{0,1} \\
b_{-1,-1} & b_{-1,0} & b_{-1,1}
\end{array}\right]
$$

respectively.

The simplest model of locally-connected networks on a plane is the one whose cell each links its three nearest neighbors, i.e. the sphere of influence 


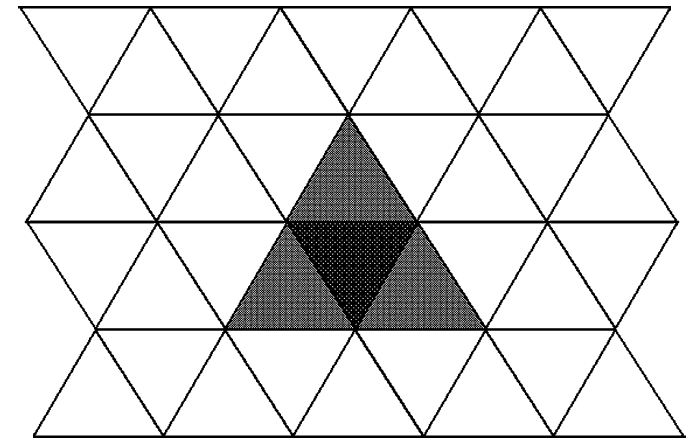

Fig. 1. Cell $C_{i}$ and its three nearest locally-connected neighbors on a plane.

of cell $C_{i}$ is a large triangle consisting of four small triangles as shown in Fig. 1.

In this situation, we can extend (4) to the following form:

$$
\frac{d x_{i}}{d t}=-x_{i}+z+a f\left(x_{i}\right)+\sum_{i=1}^{4} b_{i} u_{i}, \quad i \in Z
$$

This is equivalent as in (6), with

$$
\mathrm{B}=\left[\begin{array}{ccc}
0 & b_{1} & 0 \\
0 & b_{2} & 0 \\
b_{3} & 0 & b_{4}
\end{array}\right]
$$

We only consider the binary input-output operations of (7) in this paper.

Let

$$
\begin{array}{r}
U=\left\{u=\left(u_{1}, u_{2}, u_{3}, u_{4}\right)^{T} \mid u_{i} \in\{-1,1\},\right. \\
i=1,2,3,4\}
\end{array}
$$

where $U$ is a set of binary input vectors of (7), with

$$
U=\{-1,1\}^{4}=\left\{u^{k} \mid k=0,1,2, \ldots, 15\right\}
$$

in which

$$
k=\bar{u}_{1} 2^{3}+\bar{u}_{2} 2^{2}+\bar{u}_{3} 2+\bar{u}_{4}, \quad \bar{u}_{i}= \begin{cases}1 & \text { if } u_{i}=1 \\ 0 & \text { if } u_{i}=-1\end{cases}
$$

Namely,

$$
\begin{array}{cl}
u^{0}=(-1,-1,-1,-1)^{T}, & u^{1}=(-1,-1,-1,1)^{T}, \\
u^{2}=(-1,-1,1,-1)^{T}, & u^{3}=(-1,-1,1,1)^{T}, \\
u^{4}=(-1,1,-1,-1)^{T}, & u^{5}=(-1,1,-1,1)^{T}, \\
u^{6}=(-1,1,1,-1)^{T}, & u^{7}=(-1,1,1,1)^{T}, \\
u^{8}=(1,-1,-1,-1)^{T}, & u^{9}=(1,-1,-1,1)^{T}, \\
u^{10}=(1,-1,1,-1)^{T}, & u^{11}=(1,-1,1,1)^{T}, \\
u^{12}=(1,1,-1,-1)^{T}, & u^{13}=(1,1,-1,1)^{T}, \\
u^{14}=(1,1,1,-1)^{T}, & u^{15}=(1,1,1,1)^{T} .
\end{array}
$$

Lemma 1. (a) A subset of $U, V=\left\{u^{0}, u^{1}, u^{2}, u^{4}\right\}$, is a linearly independent subset of the binary input vector set $U$. (b) Each vector of $U$ can be linearly expressed by the vectors of $V$, i.e. $V$ is a basis of the input vector set $U$.

Proof

(a) It can be easily seen that the matrix $A$, which consists of the vectors $u^{0}, u^{1}, u^{2}$ and $u^{4}$, is invertible, where

$$
A=\left(\begin{array}{rrrr}
-1 & -1 & -1 & -1 \\
-1 & -1 & -1 & 1 \\
-1 & -1 & 1 & -1 \\
-1 & 1 & -1 & -1
\end{array}\right)
$$

and

$$
A^{-1}=\left(\begin{array}{rccc}
0.5 & -0.5 & -0.5 & -0.5 \\
-0.5 & 0 & 0 & 0.5 \\
-0.5 & 0 & 0.5 & 0 \\
-0.5 & 0.5 & 0 & 0
\end{array}\right)
$$

Thus, $V=\left\{u^{0}, u^{1}, u^{2}, u^{4}\right\}$ is a linearly independent group of $U$.

(b) From (11), it is easy to see that

$$
\begin{gathered}
u^{3}=-u^{0}+u^{1}+u^{2}, \quad u^{5}=-u^{0}+u^{1}+u^{4}, \\
u^{6}=-u^{0}+u^{2}+u^{4}, \\
u^{7}=-2 u^{0}+u^{1}+u^{2}+u^{4}, \\
u^{8}=2 u^{0}-u^{1}-u^{2}-u^{4}, \quad u^{9}=u^{0}-u^{2}-u^{4}, \\
u^{10}=u^{0}-u^{1}-u^{4}, \quad u^{11}=-u^{4}, \\
u^{12}=u^{0}-u^{1}-u^{2}, \quad u^{13}=-u^{2}, \\
u^{14}=-u^{1}, \quad u^{15}=-u^{0} .
\end{gathered}
$$

The proof of the Lemma is thus completed.

Next, let

$$
\begin{aligned}
w_{k} & =z+\sum_{i=1}^{4} b_{i} u_{i}^{k} \\
& =z+\left(u_{1}^{k}, u_{2}^{k}, u_{3}^{k}, u_{4}^{k}\right)\left(\begin{array}{c}
b_{1} \\
b_{2} \\
b_{3} \\
b_{4}
\end{array}\right)
\end{aligned}
$$

Here, $w_{k}$ is called the offset level of the CNN (7) with respect to the input vector $u^{k}(k=0,1,2, \ldots$, 15) [Chua, 1999]. 
Lemma 2. Assume $a>1$. If, for all $u^{k} \in U(k=$ $0,1,2,3, \ldots, 15)$,

$$
\left|w_{k}\right|>a-1
$$

then the CNN (7) has a constant steady-state output $y_{i}(+\infty)=\lim _{t \rightarrow+\infty} y_{i}(t)$ for each cell $C_{i}$, which is independent of the initial state $x_{i}(0)$ and can be expressed in terms of the constant binary input $u^{k} \in U$ via the following formula:

$$
y_{i}(+\infty)=\operatorname{sgn}\left(w_{k}\right), \quad(k=0,1,2,3, \ldots, 15) .
$$

Proof. Because the DP plot (an acronym for the driving-point plot [Chua et al., 1985]) of $\dot{x}_{i}=$ $-x_{i}+a f\left(x_{i}\right)+w_{k}$ depends only on two parameters, namely, the self-feedback coefficient $a$ and the offset level $w_{k}$, the proof is similar to that of Theorem 2.8.1 in [Chua, 1999]. Also, $y_{i}(+\infty)$, the output of $x_{i}$, depends only on the sign of $w_{k}$. For simplicity, we omit the details.

Theorem 1. For the uncoupled $C N N$ (7), we have the following:

(1) the following relations among $A$ in (12), consisting of $V=\left\{u^{0}, u^{1}, u^{2}, u^{4}\right\}$, the basis of $U$, the offset levels $w_{k}(k=0,1,2,4)$, and the threshold $z$, where

$$
A\left(\begin{array}{l}
b_{1} \\
b_{2} \\
b_{3} \\
b_{4}
\end{array}\right)=\left(\begin{array}{l}
w_{0}-z \\
w_{1}-z \\
w_{2}-z \\
w_{4}-z
\end{array}\right)
$$

or

$$
\left(\begin{array}{l}
b_{1} \\
b_{2} \\
b_{3} \\
b_{4}
\end{array}\right)=A^{-1}\left(\begin{array}{l}
w_{0}-z \\
w_{1}-z \\
w_{2}-z \\
w_{4}-z
\end{array}\right)
$$

(2) the offset levels $w_{k}$ of (7), except $w_{0}, w_{1}, w_{2}$ and $w_{4}$, given by

$$
\begin{aligned}
w_{3}=-w_{0}+w_{1}+w_{2}, & w_{5}=-w_{0}+w_{1}+w_{4}, \\
w_{6}=-w_{0}+w_{2}+w_{4}, & w_{7}=-w_{0}+w_{3}+w_{4}, \\
w_{8}=2 z-w_{7}, & w_{9}=2 z-w_{6}, \\
w_{10}=2 z-w_{5}, & w_{11}=2 z-w_{4}, \\
w_{12}=2 z-w_{3}, & w_{13}=2 z-w_{2}, \\
w_{14}=2 z-w_{1}, & w_{15}=2 z-w_{0} .
\end{aligned}
$$

Proof. (1) The formula (18) or (19) can be directly obtained from (15).
(2) From (14) and (15), we have

$$
\begin{aligned}
w_{3} & =z+u^{3}\left(\begin{array}{l}
b_{1} \\
b_{2} \\
b_{3} \\
b_{4}
\end{array}\right) \\
& =z+\left(-u^{0}+u^{1}+u^{2}\right)\left(\begin{array}{l}
b_{1} \\
b_{2} \\
b_{3} \\
b_{4}
\end{array}\right) \\
& =z-\left(w_{0}-z\right)+\left(w_{1}-z\right)+\left(w_{2}-z\right) \\
& =-w_{0}+w_{1}+w_{2}
\end{aligned}
$$

and the calculations of $w_{5}, w_{6}$ and $w_{7}$ are similar to $w_{3}$. As to $w_{8}$, from (14) and (15), we have $u^{8}=-u^{7}$ and

$$
\begin{aligned}
& w_{8}=z+u^{8}\left(\begin{array}{l}
b_{1} \\
b_{2} \\
b_{3} \\
b_{4}
\end{array}\right)=z-u^{7}\left(\begin{array}{l}
b_{1} \\
b_{2} \\
b_{3} \\
b_{4}
\end{array}\right) \\
&=z-\left(w_{7}-z\right)=2 z-w_{7} .
\end{aligned}
$$

Similarly, we obtain other $w_{k}(k=9,10, \ldots, 15)$ as shown in (20). The proof is thus completed.

Each binary input vector $u^{k}$ of $U$ is called a Boolean window.

If its corresponding binary output is $v_{k}, v_{k} \in$ $\{-1,1\}$, then every truth table shown in Table 1 is equivalent to a Boolean function or a Boolean CNN gene. Obviously, there are $2^{2^{4}}=65536$ different Boolean-function truth tables of four variables.

From Lemma 2 and Theorem 1, we know that if $\left|w_{k}\right|>a-1>0$ then the truth table of the inputoutput operation of CNN (7) can be obtained as shown in Table 2.

Thus, applying Theorem 1, we can immediately get the following result.

Theorem 2. A Boolean function $F\left(u^{k}\right)=v_{k}(k=$ $0,1,2, \ldots, 15)$ is linearly separable if, and only if, there exist constants $w_{0}, w_{1}, w_{2}, w_{4}$ and $z$ such that $v_{k}=\operatorname{sgn}\left(w_{k}\right)\left(w_{k} \neq 0, \quad k=0,1,2, \ldots, 15\right)$, where $w_{k}$ satisfies formulas (20) in Theorem 1.

Theorems 1 and 2 will be the most important results for realizing Boolean genes via the uncoupled CNN (7), as further discussed in the next section. 
Table 1. Boolean-function truth table of four variables.

\begin{tabular}{rcc}
\hline$k$ & Boolean Window & Output Pattern \\
\hline 0 & $u^{0}$ & $v_{0}$ \\
1 & $u^{1}$ & $v_{2}$ \\
3 & $u^{3}$ & $v_{3}$ \\
4 & $u^{4}$ & $v_{4}$ \\
5 & $u^{5}$ & $v_{5}$ \\
6 & $u^{6}$ & $v_{6}$ \\
7 & $u^{7}$ & $v_{7}$ \\
8 & $u^{8}$ & $v_{8}$ \\
9 & $u^{9}$ & $v_{9}$ \\
10 & $u^{10}$ & $v_{10}$ \\
11 & $u^{11}$ & $v_{11}$ \\
12 & $u^{12}$ & $v_{12}$ \\
13 & $u^{13}$ & $v_{13}$ \\
14 & $u^{14}$ & $v_{14}$ \\
15 & $u^{15}$ & $v_{15}$ \\
\hline
\end{tabular}

Table 2. Truth table of the input-output operations of CNN (7).

\begin{tabular}{cccc}
\hline & Boolean & Output & \\
$k$ & Window & Pattern & $w_{k}$ \\
\hline 0 & $u^{0}$ & $\operatorname{sgn}\left(w_{0}\right)$ & $w_{0}$ any given \\
1 & $u^{1}$ & $\operatorname{sgn}\left(w_{1}\right)$ & $w_{1}$ any given \\
2 & $u^{2}$ & $\operatorname{sgn}\left(w_{2}\right)$ & $w_{2}$ any given \\
3 & $u^{3}$ & $\operatorname{sgn}\left(w_{3}\right)$ & $w_{3}=w_{1}+w_{2}-w_{0}$ \\
4 & $u^{4}$ & $\operatorname{sgn}\left(w_{4}\right)$ & $w_{4}$ any given \\
5 & $u^{5}$ & $\operatorname{sgn}\left(w_{5}\right)$ & $w_{5}=w_{1}+w_{4}-w_{0}$ \\
6 & $u^{6}$ & $\operatorname{sgn}\left(w_{6}\right)$ & $w_{6}=w_{2}+w_{4}-w_{0}$ \\
7 & $u^{7}$ & $\operatorname{sgn}\left(w_{7}\right)$ & $w_{7}=w_{3}+w_{4}-w_{0}$ \\
8 & $u^{8}$ & $\operatorname{sgn}\left(w_{8}\right)$ & $w_{8}=2 z-w_{7}$ \\
9 & $u^{9}$ & $\operatorname{sgn}\left(w_{9}\right)$ & $w_{9}=2 z-w_{6}$ \\
10 & $u^{10}$ & $\operatorname{sgn}\left(w_{10}\right)$ & $w_{10}=2 z-w_{5}$ \\
11 & $u^{11}$ & $\operatorname{sgn}\left(w_{11}\right)$ & $w_{11}=2 z-w_{4}$ \\
12 & $u^{12}$ & $\operatorname{sgn}\left(w_{12}\right)$ & $w_{12}=2 z-w_{3}$ \\
13 & $u^{13}$ & $\operatorname{sgn}\left(w_{13}\right)$ & $w_{13}=2 z-w_{2}$ \\
14 & $u^{14}$ & $\operatorname{sgn}\left(w_{14}\right)$ & $w_{14}=2 z-w_{1}$ \\
15 & $u^{15}$ & $\operatorname{sgn}\left(w_{15}\right)$ & $w_{15}=2 z-w_{0}$ \\
\hline
\end{tabular}

\section{Realization and Bifurcation of Boolean Functions}

Firstly, we present some elementary Lemmas.

Lemma 3. For any three different real numbers $w_{0}, w_{1}$ and $w_{2}$, let $w_{3}=w_{1}+w_{2}-w_{0}$. Then, all the allowable orders of the four numbers $w_{0}, w_{1}, w_{2}$ and $w_{3}$ are listed as follows:
(a) $w_{0}<w_{1}<w_{2}<w_{3}$,
(b) $w_{0}<w_{2}<w_{1}<w_{3}$,
(c) $w_{2}<w_{0}<w_{3}<w_{1}$,
(d) $w_{2}<w_{3}<w_{0}<w_{1}$,
(e) $w_{1}<w_{0}<w_{3}<w_{2}$,
(f) $w_{1}<w_{3}<w_{0}<w_{2}$,
(g) $w_{3}<w_{1}<w_{2}<w_{0}$,
(h) $w_{3}<w_{2}<w_{1}<w_{0}$,

or, in a simple form,

$$
w_{i_{0}}<w_{i_{1}}<w_{i_{2}}<w_{i_{3}},
$$

where $\left(i_{0}, i_{1}, i_{2}, i_{3}\right)$ are

$$
\begin{array}{ll}
\text { (a) }(0,1,2,3), & (b)(0,2,1,3), \\
\text { (c) }(2,0,3,1), & (d)(2,3,0,1), \\
(e)(1,0,3,2), & (f)(1,3,0,2), \\
\text { (g) }(3,1,2,0), & (h)(3,2,1,0),
\end{array}
$$

respectively.

Lemma 4. For any three different real numbers $w_{0}, w_{1}, w_{2}$, let $w_{3}=w_{1}+w_{2}-w_{0}$ and for any $w_{4}, w_{4} \neq w_{j}(j=0,1,2,3)$, let $w_{5}=w_{4}+w_{1}-$ $w_{0}, w_{6}=w_{4}+w_{2}-w_{0}, w_{7}=w_{4}+w_{3}-w_{0}$. Then the eight numbers $w_{k}(k=0,1,2, \ldots, 7)$ satisfy the following two properties:

\section{Property A}

$$
w_{0}+w_{7}=w_{1}+w_{6}=w_{2}+w_{5}=w_{3}+w_{4}
$$

and for any allowable ordering of $w_{k}(k=0,1$, $2, \ldots, 7)$, namely,

$$
w_{i_{0}}<w_{i_{1}}<w_{i_{2}}<w_{i_{3}}<w_{i_{4}}<w_{i_{5}}<w_{i_{6}}<w_{i_{7}},
$$

we have

$$
w_{i_{0}}+w_{i_{7}}=w_{i_{1}}+w_{i_{6}}=w_{i_{2}}+w_{i_{5}}=w_{i_{3}}+w_{i_{4}}
$$

and

$$
i_{0}+i_{7}=i_{1}+i_{6}=i_{2}+i_{5}=i_{3}+i_{4}=7,
$$

where $\left(i_{0}, i_{1}, i_{2}, i_{3}, i_{4}, i_{5}, i_{6}, i_{7}\right)$ is an arrangement of $(0,1,2,3,4,5,6,7)$.

Property B. There are 96 different kinds of allowable orderings of the eight numbers $w_{k}(k=0,1,2$, $3,4,5,6,7)$, namely,

(a) if $w_{0}<w_{1}<w_{2}<w_{3}$, then $w_{4}<w_{5}<$ $w_{6}<w_{7}$, and all the allowable orderings of eight numbers $w_{0}, w_{1}, w_{2}, w_{3}, w_{4}, w_{5}, w_{6}$ and $w_{7}$ are as follows:

$$
w_{i_{0}}<w_{i_{1}}<w_{i_{2}}<w_{i_{3}}<w_{i_{4}}<w_{i_{5}}<w_{i_{6}}<w_{i_{7}},
$$


where $\left(i_{0}, i_{1}, i_{2}, i_{3}, i_{4}, i_{5}, i_{6}, i_{7}\right)$ are
(1) $(0,1,2,3,4,5,6,7)$,
(2) $(0,1,2,4,3,5,6,7)$,
(3) $(0,1,4,2,5,3,6,7)$,
(4) $(0,4,1,5,2,6,3,7)$,
(5) $(4,0,5,1,6,2,7,3)$,
(6) $(4,5,0,6,1,7,2,3)$,
(7) $(4,5,6,0,7,1,2,3)$,
(8) $(4,5,6,7,0,1,2,3)$,
(9) $(0,1,4,5,2,3,6,7)$,
(10) $(0,4,1,2,5,6,3,7)$,
(11) $(4,0,5,6,1,2,7,3)$,
(12) $(4,5,0,1,6,7,2,3)$,

(25)

respectively;

(b) if $w_{0}<w_{2}<w_{1}<w_{3}$, then $w_{4}<w_{6}<$ $w_{5}<w_{7}$, and all the allowable orderings of eight numbers $w_{0}, w_{1}, w_{2}, w_{3}, w_{4}, w_{5}, w_{6}$ and $w_{7}$ are as follows:

$w_{i_{0}}<w_{i_{1}}<w_{i_{2}}<w_{i_{3}}<w_{i_{4}}<w_{i_{5}}<w_{i_{6}}<w_{i_{7}}$, where $\left(i_{0}, i_{1}, i_{2}, i_{3}, i_{4}, i_{5}, i_{6}, i_{7}\right)$ are
(13) $(0,2,1,3,4,6,5,7)$,
(14) $(0,2,1,4,3,6,5,7)$,
(15) $(0,2,4,1,6,3,5,7)$,
(16) $(0,4,2,6,1,5,3,7)$,
(17) $(4,0,6,2,5,1,7,3)$,
(18) $(4,6,0,5,2,7,1,3)$,
(19) $(4,6,5,0,7,2,1,3)$,
(20) $(4,6,5,7,0,2,1,3)$,
(21) $(0,2,4,6,1,3,5,7)$,
(22) $(0,4,2,1,6,5,3,7)$,
(23) $(4,0,6,5,2,1,7,3)$,
(24) $(4,6,0,2,5,7,1,3)$,

$(26)$

respectively;

(c) if $w_{2}<w_{0}<w_{3}<w_{1}$, then $w_{6}<w_{4}<$ $w_{7}<w_{5}$, and all the allowable orderings of eight numbers $w_{0}, w_{1}, w_{2}, w_{3}, w_{4}, w_{5}, w_{6}$ and $w_{7}$ are as follows:

$w_{i_{0}}<w_{i_{1}}<w_{i_{2}}<w_{i_{3}}<w_{i_{4}}<w_{i_{5}}<w_{i_{6}}<w_{i_{7}}$, where $\left(i_{0}, i_{1}, i_{2}, i_{3}, i_{4}, i_{5}, i_{6}, i_{7}\right)$ are

(25) $(2,0,3,1,6,4,7,5), \quad(26)(2,0,3,6,1,4,7,5)$,

(27) $(2,0,6,3,4,1,7,5), \quad(28)(2,6,0,4,3,7,1,5)$,

(29) $(6,2,4,0,7,3,5,1), \quad(30)(6,4,2,7,0,5,3,1)$,

(31) $(6,4,7,2,5,0,3,1), \quad(32)(6,4,7,5,2,0,3,1)$,

(33) $(2,0,6,4,3,1,7,5), \quad(34)(2,6,0,3,4,7,1,5)$,

(35) $(6,2,4,7,0,3,5,1), \quad(36)(6,4,2,0,7,5,3,1)$,

respectively;

(d) if $w_{2}<w_{3}<w_{0}<w_{1}$, then $w_{6}<w_{7}<$ $w_{4}<w_{5}$, and all the allowable orderings of eight numbers $w_{0}, w_{1}, w_{2}, w_{3}, w_{4}, w_{5}, w_{6}$ and $w_{7}$ are as follows:

$w_{i_{0}}<w_{i_{1}}<w_{i_{2}}<w_{i_{3}}<w_{i_{4}}<w_{i_{5}}<w_{i_{6}}<w_{i_{7}}$, where $\left(i_{0}, i_{1}, i_{2}, i_{3}, i_{4}, i_{5}, i_{6}, i_{7}\right)$ are

(37) $(2,3,0,1,6,7,4,5), \quad(38)(2,3,0,6,1,7,4,5)$,

(39) $(2,3,6,0,7,1,4,5), \quad(40)(2,6,3,7,0,4,1,5)$,

(41) $(6,2,7,3,4,0,5,1), \quad(42)(6,7,2,4,3,5,0,1)$,

(43) $(6,7,4,2,5,3,0,1), \quad(44)(6,7,4,5,2,3,0,1)$,

(45) $(2,3,6,7,0,1,4,5), \quad(46)(2,6,3,0,7,4,1,5)$,

$(47)(6,2,7,4,3,0,5,1), \quad(48)(6,7,2,3,4,5,0,1)$,

(28)

respectively;

(e) if $w_{1}<w_{0}<w_{3}<w_{2}$, then $w_{5}<w_{4}<$ $w_{7}<w_{6}$, and all the allowable orderings of eight numbers $w_{0}, w_{1}, w_{2}, w_{3}, w_{4}, w_{5}, w_{6}$ and $w_{7}$ are as follows:

$w_{i_{0}}<w_{i_{1}}<w_{i_{2}}<w_{i_{3}}<w_{i_{4}}<w_{i_{5}}<w_{i_{6}}<w_{i_{7}}$,

where $\left(i_{0}, i_{1}, i_{2}, i_{3}, i_{4}, i_{5}, i_{6}, i_{7}\right)$ are

(49) $(1,0,3,2,5,4,7,6), \quad(50)(1,0,3,5,2,4,7,6)$,

(51) $(1,0,5,3,4,2,7,6), \quad(52)(1,5,0,4,3,7,2,6)$,

(53) $(5,1,4,0,7,3,6,2), \quad(54)(5,4,1,7,0,6,3,2)$,

$(55)(5,4,7,1,6,0,3,2), \quad(56)(5,4,7,6,1,0,3,2)$,

(57) $(1,0,5,4,3,2,7,6), \quad(58)(1,5,0,3,4,7,2,6)$,

(59) $(5,1,4,7,0,3,6,2), \quad(60)(5,4,1,0,7,6,3,2)$,

respectively;

(f) if $w_{1}<w_{3}<w_{0}<w_{2}$, then $w_{5}<w_{7}<$ $w_{4}<w_{6}$, and all the allowable orderings of eight numbers $w_{0}, w_{1}, w_{2}, w_{3}, w_{4}, w_{5}, w_{6}$ and $w_{7}$ are as follows:

$w_{i_{0}}<w_{i_{1}}<w_{i_{2}}<w_{i_{3}}<w_{i_{4}}<w_{i_{5}}<w_{i_{6}}<w_{i_{7}}$, where $\left(i_{0}, i_{1}, i_{2}, i_{3}, i_{4}, i_{5}, i_{6}, i_{7}\right)$ are

(61) $(1,3,0,2,5,7,4,6), \quad(62)(1,3,0,5,2,7,4,6)$,

(63) $(1,3,5,0,7,2,4,6), \quad(64)(1,5,3,7,0,4,2,6)$,

(65) $(5,1,7,3,4,0,6,2), \quad(66)(5,7,1,4,3,6,0,2)$,

(67) $(5,7,4,1,6,3,0,2), \quad(68)(5,7,4,6,1,3,0,2)$,

(69) $(1,3,5,7,0,2,4,6), \quad(70)(1,5,3,0,7,4,2,6)$,

(71) $(5,1,7,4,3,0,6,2), \quad(72)(5,7,1,3,4,6,0,2)$,

$(30)$

respectively;

(g) if $w_{3}<w_{1}<w_{2}<w_{0}$, then $w_{7}<w_{5}<$ $w_{6}<w_{4}$, and all the allowable orderings of eight 
numbers $w_{0}, w_{1}, w_{2}, w_{3}, w_{4}, w_{5}, w_{6}$ and $w_{7}$ are as follows:

$$
w_{i_{0}}<w_{i_{1}}<w_{i_{2}}<w_{i_{3}}<w_{i_{4}}<w_{i_{5}}<w_{i_{6}}<w_{i_{7}},
$$

where $\left(i_{0}, i_{1}, i_{2}, i_{3}, i_{4}, i_{5}, i_{6}, i_{7}\right)$ are
(73) $(3,1,2,0,7,5,6,4)$,
(74) $(3,1,2,7,0,5,6,4)$,
(75) $(3,1,7,2,5,0,6,4)$,
(76) $(3,7,1,5,2,6,0,4)$,
(77) $(7,3,5,1,6,2,4,0)$,
(78) $(7,5,3,6,1,4,2,0)$,
(79) $(7,5,6,3,4,1,2,0)$,
(80) $(7,5,6,4,3,1,2,0)$,
(81) $(3,1,7,5,2,0,6,4)$,
(82) $(3,7,1,2,5,6,0,4)$,
(83) $(7,3,5,6,1,2,4,0)$,
(84) $(7,5,3,1,6,4,2,0)$,

\section{respectively;}

(h) if $w_{3}<w_{2}<w_{1}<w_{0}$, then $w_{7}<w_{6}<$ $w_{5}<w_{4}$, and all the allowable orderings of eight numbers $w_{0}, w_{1}, w_{2}, w_{3}, w_{4}, w_{5}, w_{6}$ and $w_{7}$ are as follows:

$$
w_{i_{0}}<w_{i_{1}}<w_{i_{2}}<w_{i_{3}}<w_{i_{4}}<w_{i_{5}}<w_{i_{6}}<w_{i_{7}},
$$

where $\left(i_{0}, i_{1}, i_{2}, i_{3}, i_{4}, i_{5}, i_{6}, i_{7}\right)$ are

$$
\begin{array}{ll}
(85)(3,2,1,0,7,6,5,4), & (86)(3,2,1,7,0,6,5,4), \\
(87)(3,2,7,1,6,0,5,4), & (88)(3,7,2,6,1,5,0,4), \\
(89)(7,3,6,2,5,1,4,0), & (90)(7,6,3,5,2,4,1,0), \\
(91)(7,6,5,3,4,2,1,0), & (92)(7,6,5,4,3,2,1,0), \\
(93)(3,2,7,6,1,0,5,4), & (94)(3,7,2,1,6,5,0,4), \\
(95)(7,3,6,5,2,1,4,0), & (96)(7,6,3,2,5,4,1,0),
\end{array}
$$

\section{respectively.}

Lemmas 3 and 4 can be easily proved, we omit them for simplicity.

Theorem 3. Nine Boolean functions can be realized via the uncoupled $C N N(7)$ by changing the threshold $z$ for any allowable ordering of the eight numbers $w_{k}(k=0,1,2,3,4,5,6,7)$ in Lemma 4, namely,

$$
w_{i_{0}}<w_{i_{1}}<w_{i_{2}}<w_{i_{3}}<w_{i_{4}}<w_{i_{5}}<w_{i_{6}}<w_{i_{7}} .
$$

Proof. Firstly, nine open intervals can be given on $(-\infty,+\infty)$ according to the numbers $w_{i_{k}}(k=$ $0,1,2,3,4,5,6,7)$; they are:

$$
\begin{array}{cl}
I_{0}=\left(-\infty, \frac{w_{i_{0}}}{2}\right), & I_{1}=\left(\frac{w_{i_{0}}}{2}, \frac{w_{i_{1}}}{2}\right), \\
I_{2}=\left(\frac{w_{i_{1}}}{2}, \frac{w_{i_{2}}}{2}\right), \quad I_{3}=\left(\frac{w_{i_{2}}}{2}, \frac{w_{i_{3}}}{2}\right),
\end{array}
$$

$$
\begin{gathered}
I_{4}=\left(\frac{w_{i_{3}}}{2}, \frac{w_{i_{4}}}{2}\right), \quad I_{5}=\left(\frac{w_{i_{4}}}{2}, \frac{w_{i_{5}}}{2}\right), \\
I_{6}=\left(\frac{w_{i_{5}}}{2}, \frac{w_{i_{6}}}{2}\right), \quad I_{7}=\left(\frac{w_{i_{6}}}{2}, \frac{w_{i_{7}}}{2}\right), \\
I_{8}=\left(\frac{w_{i_{7}}}{2},+\infty\right) .
\end{gathered}
$$

For every interval $I_{j}(j=0,1,2, \ldots, 8)$, and for any given $z_{j}, z_{j} \in I_{j}$, we can calculate $\left(b_{1}, b_{2}, b_{3}, b_{4}\right)^{T}$ by using the $w_{0}, w_{1}, w_{2}, w_{4}$ and $z_{j}$ in formula (19), as follows:

$$
\left(\begin{array}{l}
b_{1} \\
b_{2} \\
b_{3} \\
b_{4}
\end{array}\right)=A^{-1}\left(\begin{array}{l}
w_{0}-z_{j} \\
w_{1}-z_{j} \\
w_{2}-z_{j} \\
w_{4}-z_{j}
\end{array}\right),
$$

where $A^{-1}$ is the inverse matrix of $A$ in (12).

Clearly, from Lemma 4, we have

$$
\begin{aligned}
w_{3}=-w_{0}+w_{1}+w_{2}, & w_{5}=-w_{0}+w_{1}+w_{4}, \\
w_{6}=-w_{0}+w_{2}+w_{4}, & w_{7}=-w_{0}+w_{3}+w_{4}, \\
w_{8}=2 z_{j}-w_{7}, & w_{9}=2 z_{j}-w_{6}, \\
w_{10}=2 z_{j}-w_{5}, & w_{11}=2 z_{j}-w_{4}, \\
w_{12}=2 z_{j}-w_{3}, & w_{13}=2 z_{j}-w_{2}, \\
w_{14}=2 z_{j}-w_{1}, & w_{15}=2 z_{j}-w_{0} .
\end{aligned}
$$

Next, let

$$
a=1+\frac{1}{2} \min \left\{\left|w_{k}\right| \mid k=0,1,2, \ldots, 15\right\} .
$$

Then, we can construct the uncoupled CNN (7) by using the six numbers $b_{1}, b_{2}, b_{3}, b_{4}, z_{j}$ and $a$, its gene is given by

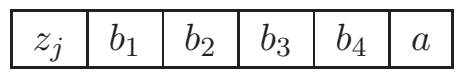

which can realize a Boolean function whose binary output pattern is

$$
\left(\operatorname{sgn}\left(w_{0}\right), \operatorname{sgn}\left(w_{1}\right), \operatorname{sgn}\left(w_{2}\right), \ldots, \operatorname{sgn}\left(w_{15}\right)\right) .
$$

The proof is thus completed.

Example 1. If $w_{0}=2, w_{1}=6, w_{2}=10, w_{4}=18$, then $w_{3}=14, w_{5}=22, w_{6}=26, w_{7}=30$. Consequently, $z_{1} \in I_{1}=\left(w_{0} / 2, w_{1} / 2\right)=(1,3)$. Take 
$z_{1}=2$, the center point of the interval $I_{1}$. Then,

$$
\begin{aligned}
\left(\begin{array}{l}
b_{1} \\
b_{2} \\
b_{3} \\
b_{4}
\end{array}\right) & =A^{-1}\left(\begin{array}{l}
w_{0}-z_{1} \\
w_{1}-z_{1} \\
w_{2}-z_{1} \\
w_{4}-z_{1}
\end{array}\right)=A^{-1}\left(\begin{array}{r}
0 \\
4 \\
8 \\
16
\end{array}\right) \\
& =\left(\begin{array}{r}
-14 \\
8 \\
4 \\
2
\end{array}\right),
\end{aligned}
$$

and $w_{8}=-26, w_{9}=-22, w_{10}=-18, w_{11}=$ $-14, w_{12}=-10, w_{13}=-6, w_{14}=-2, w_{15}=2$, $a=1+(1 / 2) \min \left\{\left|w_{k}\right| \mid k=0,1,2, \ldots, 15\right\}=2$. Therefore, we can design the uncoupled CNN (7) with gene

\begin{tabular}{|l|l|l|l|l|l|}
\hline 2 & -14 & 8 & 4 & 2 & 2 \\
\hline
\end{tabular}

and output pattern

$$
\begin{aligned}
& \left(\operatorname{sgn}\left(w_{0}\right), \operatorname{sgn}\left(w_{1}\right), \ldots, \operatorname{sgn}\left(w_{15}\right)\right) \\
& \quad=(1,1,1,1,1,1,1,1, \\
& \quad-1,-1,-1,-1,-1,-1,-1,1) .
\end{aligned}
$$

Similarly, we can list the other eight genes and the corresponding output patterns, binary decoding tapes, and decimal codes of the CNN. Details are shown in Table 3.

Remark 1. If the output pattern of CNN (7) is $\left(v_{0}, v_{1}, v_{2}, \ldots, v_{15}\right)$, then its binary decoding tape is $\bar{v}_{0} \bar{v}_{1} \bar{v}_{2} \cdots \bar{v}_{15}$, where $\bar{v}_{k}=1$ if $v_{k}=1, \bar{v}_{k}=0$ if $v_{k}=-1$, and its decimal code is

$$
p=\bar{v}_{0} 2^{14}+\bar{v}_{1} 2^{13}+\cdots+\bar{v}_{14} 2+\bar{v}_{15} .
$$

We should pay more attention to the values $w_{0} / 2, w_{1} / 2, w_{2} / 2, \ldots, w_{7} / 2$ in the above theorem and example. They are the bifurcation values that yield Boolean functions. This bifurcation phenomenon is generated by changing only one parameter, i.e. the threshold $z$ of the CNN (7).

Next, we will classify all the allowable orderings of $w_{k}(k=0,1,2, \ldots, 7)$ in Lemma 4 into nine classes where the sign of $w_{k}$ is determined.

$$
\begin{aligned}
(\mathrm{I}): w_{i_{0}} & <w_{i_{1}}<w_{i_{2}}<w_{i_{3}}<w_{i_{4}} \\
& <w_{i_{5}}<w_{i_{6}}<w_{i_{7}}<0
\end{aligned}
$$

where $\left(i_{0}, i_{1}, \ldots, i_{7}\right)$ denotes all the 96 arrangements of (1) to (96) in Lemma 4;

$$
\text { (II): } \begin{aligned}
w_{i_{0}} & <w_{i_{1}}<w_{i_{2}}<w_{i_{3}}<w_{i_{4}} \\
& <w_{i_{5}}<w_{i_{6}}<0<w_{i_{7}}
\end{aligned}
$$

where $\left(i_{0}, i_{1}, \ldots, i_{7}\right)$ denotes all the 96 arrangements of (1) to (96) in Lemma 4;

$$
\text { (III): } \begin{aligned}
w_{i_{0}} & <w_{i_{1}}<w_{i_{2}}<w_{i_{3}}<w_{i_{4}} \\
& <w_{i_{5}}<0<w_{i_{6}}<w_{i_{7}}
\end{aligned}
$$

\begin{tabular}{|c|c|c|c|c|c|c|c|c|}
\hline \multicolumn{6}{|c|}{ Gene } & Output Pattern & Binary Code Tape & Decimal Code \\
\hline 16 & 0 & 8 & 4 & 2 & 2 & $(1,1,1,1,1,1,1,1,1,1,1,1,1,1,1,1)$ & 1111111111111111 & 65535 \\
\hline 14 & -2 & 8 & 4 & 2 & 2 & $(1,1,1,1,1,1,1,1,-1,1,1,1,1,1,1,1)$ & 1111111101111111 & 65407 \\
\hline 12 & -4 & 8 & 4 & 2 & 2 & $(1,1,1,1,1,1,1,1,-1,-1,1,1,1,1,1,1)$ & 1111111100111111 & 65343 \\
\hline 10 & -6 & 8 & 4 & 2 & 2 & $(1,1,1,1,1,1,1,1,-1,-1,-1,1,1,1,1,1)$ & 1111111100011111 & 65311 \\
\hline 8 & -8 & 8 & 4 & 2 & 2 & $(1,1,1,1,1,1,1,1,-1,-1,-1,-1,1,1,1,1)$ & 1111111100001111 & 65295 \\
\hline 6 & -10 & 8 & 4 & 2 & 2 & $(1,1,1,1,1,1,1,1,-1,-1,-1,-1,-1,1,1,1)$ & 1111111100000111 & 65287 \\
\hline 4 & -12 & 8 & 4 & 2 & 2 & $(1,1,1,1,1,1,1,1,-1,-1,-1,-1,-1,-1,1,1)$ & 1111111100000011 & 65283 \\
\hline 2 & -14 & 8 & 4 & 2 & 2 & $(1,1,1,1,1,1,1,1,-1,-1,-1,-1,-1,-1,-1,1)$ & 1111111100000001 & 65281 \\
\hline 0 & -16 & 8 & 4 & 2 & 2 & $(1,1,1,1,1,1,1,1,-1,-1,-1,-1,-1,-1,-1,-1)$ & 1111111100000000 & 65280 \\
\hline
\end{tabular}

where $\left(i_{0}, i_{1}, \ldots, i_{7}\right)$ denotes all the 96 arrangements of (1) to (96) in Lemma 4;

$$
\begin{aligned}
(\mathrm{IV}): w_{i_{0}} & <w_{i_{1}}<w_{i_{2}}<w_{i_{3}}<w_{i_{4}} \\
& <0<w_{i_{5}}<w_{i_{6}}<w_{i_{7}}
\end{aligned}
$$

where $\left(i_{0}, i_{1}, \ldots, i_{7}\right)$ denotes all the 96 arrangements of (1) to (96) in Lemma 4;

$$
\begin{aligned}
(\mathrm{V}): w_{i_{0}} & <w_{i_{1}}<w_{i_{2}}<w_{i_{3}}<0 \\
& <w_{i_{4}}<w_{i_{5}}<w_{i_{6}}<w_{i_{7}}
\end{aligned}
$$

Table 3. Genes, output patterns, binary decoding tapes and decimal codes of Example 1. 
where $\left(i_{0}, i_{1}, \ldots, i_{7}\right)$ denotes all the 96 arrangements of (1) to (96) in Lemma 4;

$$
\begin{aligned}
(\mathrm{VI}): w_{i_{0}} & <w_{i_{1}}<w_{i_{2}}<0<w_{i_{3}} \\
& <w_{i_{4}}<w_{i_{5}}<w_{i_{6}}<w_{i_{7}}
\end{aligned}
$$

where $\left(i_{0}, i_{1}, \ldots, i_{7}\right)$ denotes all the 96 arrangements of (1) to (96) in Lemma 4;

$$
\begin{aligned}
(\mathrm{VII}): w_{i_{0}} & <w_{i_{1}}<0<w_{i_{2}}<w_{i_{3}} \\
& <w_{i_{4}}<w_{i_{5}}<w_{i_{6}}<w_{i_{7}}
\end{aligned}
$$

where $\left(i_{0}, i_{1}, \ldots, i_{7}\right)$ denotes all the 96 arrangements of (1) to (96) in Lemma 4;

$$
\text { (VIII): } \begin{aligned}
w_{i_{0}} & <0<w_{i_{1}}<w_{i_{2}}<w_{i_{3}} \\
& <w_{i_{4}}<w_{i_{5}}<w_{i_{6}}<w_{i_{7}}
\end{aligned}
$$

where $\left(i_{0}, i_{1}, \ldots, i_{7}\right)$ denotes all the 96 arrangements of (1) to (96) in Lemma 4;

$$
\text { (IX): } \begin{aligned}
0 & <w_{i_{0}}<w_{i_{1}}<w_{i_{2}}<w_{i_{3}} \\
& <w_{i_{4}}<w_{i_{5}}<w_{i_{6}}<w_{i_{7}}
\end{aligned}
$$

where $\left(i_{0}, i_{1}, \ldots, i_{7}\right)$ denotes all the 96 arrangements of (1) to (96) in Lemma 4.

Lemma 5. For every one of the above ordering classes of $w_{k}(k=0,1,2, \ldots, 7)$, property $A$ of Lemma 4 holds. Moreover,

(i) for a pair of allowable orders (a) and (b) in the same class, if the order (b) is obtained by exchanging one pair of numbers of (a), i.e.

$$
\left\{\begin{aligned}
(\mathrm{a}) w_{i_{0}} & <w_{i_{1}}<w_{i_{2}}<w_{i_{3}}<w_{i_{4}} \\
& <w_{i_{5}}<w_{i_{6}}<w_{i_{7}} \\
\text { (b) } w_{i_{0}} & <w_{i_{1}}<w_{i_{2}}<\underline{w_{i_{4}}<w_{i_{3}}} \\
& <w_{i_{5}}<w_{i_{6}}<w_{i_{7}}
\end{aligned}\right.
$$

then, according to Theorem 3, (b) can only realize a new Boolean function that is different from the Boolean functions yielded by (a). This situation is denoted by

$$
\text { (a) } \frac{1}{(b) ;}
$$

(ii) for a pair of allowable orders (a) and (b) in the same class, if the order (b) is obtained by exchanging two pairs of numbers of (a), i.e.

$$
\left\{\begin{aligned}
\text { (a) } w_{i_{0}} & <w_{i_{1}}<w_{i_{2}}<w_{i_{3}}<w_{i_{4}} \\
& <w_{i_{5}}<w_{i_{6}}<w_{i_{7}} \\
\text { (b) } w_{i_{0}} & <w_{i_{1}}<\underline{w_{i_{3}}<w_{i_{2}}}<\underline{w_{i_{5}}<w_{i_{4}}} \\
& <w_{i_{6}}<w_{i_{7}}
\end{aligned}\right.
$$

or

$$
\left\{\begin{aligned}
\text { (a) } w_{i_{0}} & <w_{i_{1}}<w_{i_{2}}<w_{i_{3}}<w_{i_{4}} \\
& <w_{i_{5}}<w_{i_{6}}<w_{i_{7}} \\
\text { (b) } w_{i_{0}} & <\underline{w_{i_{2}}<w_{i_{1}}}<w_{i_{3}}<w_{i_{4}} \\
& <\underline{w_{i_{6}}<w_{i_{5}}}<w_{i_{7}}
\end{aligned}\right.
$$

then, according to Theorem 3, (b) can only realize two new Boolean functions that are different from the Boolean functions yielded by (a). This situation is denoted by

$$
\text { (a) } \frac{2}{(b) ; ~}
$$

(iii) for a pair of allowable orders (a) and (b) in the same class, if the order (b) is obtained by exchanging three pairs of numbers of (a), i.e.

$$
\left\{\begin{aligned}
\text { (a) } w_{i_{0}} & <w_{i_{1}}<w_{i_{2}}<w_{i_{3}}<w_{i_{4}} \\
& <w_{i_{5}}<w_{i_{6}}<w_{i_{7}} \\
\text { (b) } w_{i_{0}} & <\underline{w_{i_{2}}<w_{i_{1}}}<\underline{w_{i_{4}}<w_{i_{3}}} \\
& <\underline{w_{i_{6}}<w_{i_{5}}}<w_{i_{7}}
\end{aligned}\right.
$$

then, according to Theorem 3, (b) can only realize three new Boolean functions that are different from the Boolean functions yielded by (a). This situation is denoted by

$$
\text { (a) } \frac{3}{}(\mathrm{~b}) \text {; }
$$

(iv) for a pair of allowable orders (a) and (b) in the same class, if the order (b) is obtained by exchanging four of pairs numbers of (a), i.e.

$$
\left\{\begin{aligned}
\text { (a) } w_{i_{0}} & <w_{i_{1}}<w_{i_{2}}<w_{i_{3}}<w_{i_{4}} \\
& <w_{i_{5}}<w_{i_{6}}<w_{i_{7}} \\
\text { (b) } \frac{w_{i_{1}}}{<w_{i_{0}}}<\underline{w_{i_{3}}<w_{i_{2}}}<\underline{w_{i_{5}}<w_{i_{4}}} &
\end{aligned}\right.
$$

then, according to Theorem 3, (b) can only realize four new Boolean functions that are different from the Boolean functions yielded by (a). This situation is denoted by

$$
\text { (a) } \frac{4}{(b) ; ~}
$$

(v) for a pair of allowable orders (a) and (b) in the same class, assume that they can be indicated in the following form:

$$
\left\{\begin{array}{l}
\text { (a) } w_{i_{0}}<\cdots<w_{i_{p}}<w_{i_{p+1}}<\cdots<w_{i_{7}} \\
\text { (b) } w_{j_{1}}<\cdots<w_{j_{p}}<w_{j_{p+1}}<\cdots<w_{j_{7}}
\end{array}\right.
$$


where

$$
\begin{aligned}
& \left\{i_{1}, \ldots, i_{p}\right\} \cup\left\{i_{p+1}, \ldots, i_{7}\right\} \\
& \quad=\left\{j_{1}, \ldots, j_{p}\right\} \cup\left\{j_{p+1}, \ldots, j_{7}\right\} \\
& \quad=\{0,1,2,3,4,5,6,7\}
\end{aligned}
$$

and the subscript sets $\left\{j_{1}, \ldots, j_{p}\right\}$ are a permutation of $\left\{i_{1}, \ldots, i_{p}\right\}$ and $\left\{j_{p+1}, \ldots, j_{7}\right\}$ are a permutation of $\left\{i_{p+1}, \ldots, i_{7}\right\}$, respectively, then (a) and (b) will realize the same output pattern when $z \in$ $\left(w_{i_{p}} / 2, w_{i_{p+1}} / 2\right)$ in (a) and $z \in\left(w_{j_{p}} / 2, w_{j_{p+1}} / 2\right)$ in (b).

Remark 2. It is allowable that one subscript set is an empty set.

Proof. (i) Firstly, the signs of $w_{k}(k=0,1,2, \ldots, 7)$ are completely determined in the order pair (a) and (b), because they are in the same order class (I) to (IX). If $z \in\left(w_{i_{3}} / 2, w_{i_{4}} / 2\right)$ in (a), then $\operatorname{sgn}(2 z-$ $\left.w_{i_{3}}\right)=1, \operatorname{sgn}\left(2 z-w_{i_{4}}\right)=-1$; if $z \in\left(w_{i_{4}} / 2, w_{i_{3}} / 2\right)$ in $(\mathrm{b})$, then $\operatorname{sgn}\left(2 z-w_{i_{3}}\right)=-1, \operatorname{sgn}\left(2 z-w_{i_{4}}\right)=1$; but all the signs of $\left(2 z-w_{i_{k}}\right)(k=0,1, \ldots, 7)$ are not changed regardless of (a) or (b) when $z$ belongs to the remaining eight intervals appeared in the proof of Theorem 3. Thus, only one output pattern of (b) is different from those of (a).

The proofs of (ii)-(iv) are similar.

For (v), we only note that when $z \in$ $\left(w_{i_{p}} / 2, w_{i_{p+1}} / 2\right)$ in (a) and $z \in\left(w_{j_{p}} / 2, w_{j_{p+1}} / 2\right)$ in (b), all signs of $\left(2 z-w_{i_{k}}\right)(k=0,1, \ldots, 7)$ are fixed. The proof of the Lemma is thus completed.
For convenience, we denote the linearly separable Boolean functions as LSBF, and the linearly separable Boolean genes as LSBG, in the following.

\section{Lemma 6}

(i) The ordering class (I) can realize 104 LSBF via the uncoupled CNN (7);

(ii) the ordering class (II) can realize 160 LSBF via the uncoupled $C N N(7)$;

(iii) the ordering class (III) can realize $240 \mathrm{LSBF}$ via the uncoupled $C N N(7)$;

(iv) the ordering class (IV) can realize $288 L S B F$ via the uncoupled CNN (7);

(v) the ordering class $(\mathrm{V})$ can realize 298 LSBF via the uncoupled $C N N(7)$;

(vi) the ordering class (VI) can realize 288 LSBF via the uncoupled $C N N(7)$;

(vii) the ordering class (VII) can realize $240 L S B F$ via the uncoupled $C N N(7)$;

(viii) the ordering class (VIII) can realize $160 \mathrm{LSBF}$ via the uncoupled $C N N(7)$;

(ix) the ordering class (IX) can realize 104 LSBF via the uncoupled $C N N(7)$.

Proof

(i) The class (I) is

$$
\begin{gathered}
w_{i_{0}}<w_{i_{1}}<w_{i_{2}}<w_{i_{3}}<w_{i_{4}} \\
<w_{i_{5}}<w_{i_{6}}<w_{i_{7}}<0 .
\end{gathered}
$$

If we use the notations of Lemma 5 , then there are some related chains of the orderings in Lemma 4 , namely,

$$
\text { (a) : (1) } \frac{1}{2}(2) \frac{2}{-}(3) \frac{3}{-}(4) \frac{4}{-}(5) \frac{3}{-}(6) \frac{2}{2}(7) \frac{1}{}(8) \text {, }
$$

Applying Theorem 3 and Lemma 5, we know that the order (1) can realize 9 LSBF. The other orders in the chain, (2)-(8), can yield 16 new LSBF. But other orders out of the chain, (9)-(12), cannot yield any new LSBF, because the LSBFs realized do appear in the chain. Thus, the whole chain (a) can generate 25 LSBF.

Further, we have other chains of $w_{k}$ as shown below:

$$
\begin{aligned}
& \text { (b) : (1) } \frac{2}{}(13)-(14) \frac{2}{-}(15) \frac{1}{-}(16) \frac{2}{-}(17)-(18)-(19)-(20) \text {; } \\
& \text { (c) : (13) } \frac{4}{-}(25) \frac{1}{2}(26) \frac{2}{2}(27) \frac{2}{2}(28) \frac{4}{-}(29) \frac{1}{-}(30) \frac{2}{2}(31)-(32) \text {; } \\
& \text { (d) : } \underline{(25)} \frac{2}{}(37)-(38) \frac{2}{4}(39) \frac{1}{-}(40) \frac{2}{-}(41) \frac{2}{-}(42)-(43)-(44) \text {; }
\end{aligned}
$$

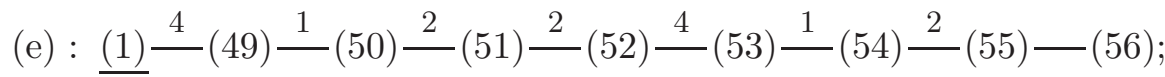




$$
\begin{aligned}
& \text { (f) : (49) } \frac{2}{2}(61)-(62) \frac{2}{-}(63) \frac{1}{-}(64) \frac{2}{2}(65) \frac{2}{2}(66)-(67)-(68) \text {; } \\
& \text { (g) : } \underline{(61)} \frac{4}{-}(73) \frac{1}{2}(74) \frac{2}{2}(75) \frac{2}{-}(76) \frac{4}{-}(77) \frac{1}{2}(78) \frac{2}{-}(79)-(80) \text {; } \\
& \text { (h) : (73) - }(85)-(86) \frac{2}{-}(87)-(88) \frac{2}{-}(89)-(90)-(91)-(92) \text {. }
\end{aligned}
$$

Remark 3. The underline above indicates that the order has appeared in the preceding orders. If no script on the midline between two orders, it implies that the latter cannot yield a new LSBF.

Applying Theorem 3 and Lemma 5 repeatedly, we can see that the chain (b) can realize 9 LSBF, the chain (c) can realize $16 \mathrm{LSBF}$, and likewise, (d) : 9, (e) :16, (f) :9, (g) : 16 and (h) : 4, respectively. Thus, the class (I) can realize a total of $104 \mathrm{LSBF}$.

(ii) Similar to (i), for class (II):

$$
w_{i_{0}}<w_{i_{1}}<w_{i_{2}}<w_{i_{3}}<w_{i_{4}}<w_{i_{5}}<w_{i_{6}}<0<w_{i_{7}} .
$$

For the pair (a) and (b) in Lemma 4, we have two related chains:

$$
\text { (4) } \frac{3}{2}(3) \frac{2}{2}(2) \frac{1}{2}(1) \frac{2}{2}(13)-(14) \frac{2}{-}(15) \frac{1}{2}(16)
$$

and

$$
\text { (5) } \frac{3}{2}(6) \frac{2}{-}(7) \frac{1}{2}(8) \frac{2}{-}(20)-(19) \frac{2}{-}(18) \frac{1}{-}(17) ;
$$

for the pair (c) and (d) in Lemma 4, we have

$$
\text { (28) } \frac{3}{2}(27) \frac{2}{2}(26) \frac{1}{2}(25) \frac{2}{-}(37)-(38) \frac{2}{-}(39) \frac{1}{-}(40)
$$

and

$$
\text { (29) } \frac{3}{3}(30) \frac{2}{2}(31) \frac{1}{3}(32) \frac{2}{2}(44)-(43) \frac{2}{-}(42) \frac{1}{3}(41) \text {; }
$$

for the pair (e) and (f) in Lemma 4, we have

$$
(52) \frac{3}{-}(51) \frac{2}{-}(50) \frac{1}{-}(49) \frac{2}{-}(61)-(62) \frac{2}{-}(63) \frac{1}{-}(64)
$$

and

$$
\text { (53) } \frac{3}{-}(54) \frac{2}{2}(55) \frac{1}{-}(56) \frac{2}{-}(68)-(67) \frac{2}{-}(66) \frac{1}{-}(65) ;
$$

for the pair (g) and (h) in Lemma 4, we have

$$
\text { (76) } \frac{3}{-}(75) \frac{2}{-}(74) \frac{1}{-}(73) \frac{2}{-}(85)-(86) \frac{2}{-}(87) \frac{1}{-}(88)
$$

and

$$
\text { (77) } \frac{3}{-}(78) \stackrel{2}{-}(79) \frac{1}{-}(80) \stackrel{2}{-}(92)-(91) \stackrel{2}{-}(90) \stackrel{1}{-}(89) .
$$

Based on Theorem 3 and Lemma 5, each pair of orders can yield 40 LSBF. Thus, the class (II) can realize exactly $160 \mathrm{LSBF}$.

Remark 4. Other orders out of the above chains cannot yield any new LSBF.

(iii) Class (III) is:

$$
w_{i_{0}}<w_{i_{1}}<w_{i_{2}}<w_{i_{3}}<w_{i_{4}}<w_{i_{5}}<0<w_{i_{6}}<w_{i_{7}}
$$


We have 12 chains of the orders shown in Lemma 4 :

$$
\begin{aligned}
& \text { (9) } \frac{1}{-}(3) \stackrel{2}{-}(2) \frac{1}{2}(1) \frac{4}{-}(49) \frac{1}{-}(50) \stackrel{2}{-}(51)-(57) \text {, } \\
& \text { (12) } \frac{1}{-}(6) \stackrel{2}{-}(7) \frac{1}{-}(8) \frac{4}{-}(56) \frac{1}{-}(55) \stackrel{2}{-}(54)-(60) \text {, } \\
& \text { (16) } \frac{1}{2}(22) \frac{2}{2}(10) \frac{1}{2}(4) \frac{4}{2}(5) \frac{1}{-}(11) \frac{2}{-}(23)-(17) \text {, } \\
& \text { (21) } \frac{1}{2}(15) \frac{2}{-}(14) \frac{1}{2}(13) \frac{4}{4}(25) \frac{1}{4}(26) \frac{2}{-}(27)-(33) \text {, } \\
& \text { (24) } \frac{1}{-}(18) \frac{2}{-}(19) \frac{1}{-}(20) \frac{4}{-}(32) \frac{1}{2}(31) \frac{2}{-}(30)-(36) \text {, } \\
& \text { (40) } \frac{1}{2}(46) \frac{2}{-}(34) \frac{1}{2}(28) \frac{4}{2}(29) \frac{1}{4}(35) \frac{2}{2}(47)-(41) \text {, } \\
& \text { (44) } \frac{1}{2}(43) \frac{2}{-}(42) \frac{1}{2}(48) \frac{4}{-}(96) \frac{1}{4}(90) \frac{2}{-}(91)-(92) \text {, } \\
& \text { (45) } \frac{1}{-}(39) \frac{2}{-}(38) \frac{1}{-}(37) \frac{4}{-}(85) \frac{1}{4}(86) \frac{2}{-}(87)-(93) \text {, } \\
& \text { (52) } \frac{1}{-}(58) \frac{2}{-}(70) \frac{1}{-}(64) \frac{4}{-}(65) \frac{1}{-}(71) \frac{2}{-}(59)-(53) \text {, } \\
& \text { (68) } \frac{1}{-}(67) \frac{2}{-}(66) \frac{1}{-}(72) \frac{4}{-}(84) \frac{1}{-}(78) \frac{2}{-}(79)-(80) \text {, } \\
& \text { (69) } \frac{1}{2}(63) \frac{2}{-}(62) \frac{1}{2}(61) \frac{4}{-}(73) \frac{1}{4}(74) \frac{2}{-}(75)-(81) \text {, } \\
& \text { (88) } \frac{1}{-}(94) \frac{2}{-}(82) \frac{1}{-}(76) \frac{4}{-}(77) \frac{1}{-}(83) \frac{2}{-}(95)-(89) \text {. }
\end{aligned}
$$

Every one of the above chains is independent. By Theorem 3 and Lemma 5, each chain can yield 20 LSBF, so that situation (III) can realize a total of $240 \mathrm{LSBF}$.

(iv) Class (IV) is:

$$
w_{i_{0}}<w_{i_{1}}<w_{i_{2}}<w_{i_{3}}<w_{i_{4}}<0<w_{i_{5}}<w_{i_{6}}<w_{i_{7}} .
$$

At this time, there are 24 independent chains of the orders shown in Lemma 4. They are:

$$
\begin{aligned}
& \text { (1) } \frac{1}{-}(2) \frac{2}{-}(14)-(13), \quad(3) \frac{3}{3}(4)-(10)-(9), \\
& \text { (5) } \frac{3}{-}(6)-(12)-(11), \quad(7) \frac{1}{3}(8) \frac{2}{-}(20)-(19), \\
& \text { (15) } \frac{3}{-}(16)-(22)-(21), \quad(17) \frac{3}{3}(18)-(24)-(23) \text {, } \\
& (25) \frac{1}{2}(26) \frac{2}{-}(38)-(37), \quad(27) \frac{3}{3}(28)-(34)-(33) \text {, } \\
& \text { (29) } \frac{3}{3}(30)-(36)-(35), \quad(31) \frac{1}{3}(32) \frac{2}{3}(44)-(43) \text {, } \\
& \text { (39) } \frac{3}{-}(40)-(46)-(45), \quad(41) \frac{3}{3}(42)-(48)-(47) \text {, } \\
& \text { (49) } \frac{1}{-}(50) \frac{2}{-}(62)-(61), \quad(51) \frac{3}{-}(52)-(58)-(57) \text {, } \\
& \text { (53) } \frac{3}{-}(54)-(60)-(59), \quad(55) \frac{1}{3}(56) \frac{2}{3}(68)-(67) \text {, } \\
& \text { (63) } \frac{3}{-}(64)-(70)-(69), \quad(65) \frac{3}{3}(66)-(72)-(71) \text {, } \\
& \text { (73) } \frac{1}{-}(74) \frac{2}{2}(86)-(85), \quad(75) \frac{3}{-}(76)-(82)-(81) \text {, }
\end{aligned}
$$




$$
\begin{array}{ll}
(77) \frac{3}{3}(78)-(84)-(83), & (79) \frac{1}{3}(80) \frac{2}{-}(92)-(91), \\
(87) \frac{3}{-}(88)-(94)-(93), & (89) \frac{3}{2}(90)-(96)-(95) .
\end{array}
$$

From Theorem 3 and Lemma 5, this situation can realize exactly 288 LSBF.

(v) Class (V) is:

$$
w_{i_{0}}<w_{i_{1}}<w_{i_{2}}<w_{i_{3}}<0<w_{i_{4}}<w_{i_{5}}<w_{i_{6}}<w_{i_{7}} .
$$

Similar to the preceding classes, there are 14 independent order chains in Lemma 4. They are:

$$
\begin{aligned}
& \text { (37) } \frac{2}{-}(25) \frac{4}{-}(13) \stackrel{2}{-}(1) \frac{4}{-}(49) \stackrel{2}{-}(61) \stackrel{4}{-}(73)-(85) \text {, } \\
& \text { (12) } \frac{2}{-}(5) \stackrel{4}{-}(4) \frac{2}{-}(9) \frac{4}{-}(57) \stackrel{2}{-}(52) \stackrel{4}{-}(53)-(60) \text {, } \\
& \text { (44) } \frac{2}{2}(32) \frac{4}{2}(20) \frac{2}{2}(8) \frac{4}{-}(56) \frac{2}{-}(68) \frac{4}{-}(80)-(92) \text {, } \\
& \text { (29) } \frac{2}{-}(36) \frac{4}{-}(24) \frac{2}{-}(17) \frac{4}{-}(16) \frac{2}{2}(21) \frac{4}{-}(33)-(28) \text {, } \\
& \text { (48) } \frac{2}{-}(41) \frac{4}{-}(40) \frac{2}{2}(45) \frac{4}{-}(93) \frac{2}{2}(88) \frac{4}{-}(89)-(96) \text {, } \\
& \text { (72) } \frac{2}{-}(65) \frac{4}{-}(64) \frac{2}{-}(69) \frac{4}{-}(81) \frac{2}{2}(76) \stackrel{4}{-}(77)-(84) \text {, } \\
& \text { (10) } \frac{2}{-}(3) \stackrel{2}{-}(2) \frac{2}{-}(14) \stackrel{2}{-}(15)-(22) \text {, } \\
& \text { (11) } \frac{2}{-}(6) \frac{2}{-}(7) \frac{2}{-}(19) \frac{2}{-}(18)-(23) \text {, } \\
& \text { (34) } \frac{2}{2}(27) \stackrel{2}{2}(26) \frac{2}{-}(38) \stackrel{2}{-}(39)-(46) \text {, } \\
& \text { (58) } \frac{2}{-}(51) \stackrel{2}{-}(50) \stackrel{2}{-}(62) \stackrel{2}{-}(63)-(70) \text {, } \\
& \text { (35) } \frac{2}{-}(30) \stackrel{2}{-}(31) \stackrel{2}{-}(43) \stackrel{2}{-}(42)-(47) \text {, } \\
& \text { (59) } \frac{2}{-}(54) \frac{2}{-}(55) \stackrel{2}{-}(67) \stackrel{2}{-}(66)-(71) \text {, } \\
& \text { (82) } \frac{2}{-}(75) \frac{2}{-}(74) \frac{2}{2}(86) \stackrel{2}{-}(87)-(94) \text {, } \\
& \text { (83) } \frac{2}{2}(78) \stackrel{2}{-}(79) \stackrel{2}{-}(91) \stackrel{2}{-}(90)-(95) \text {. }
\end{aligned}
$$

Thus, these chains can yield exactly 298 LSBF according to Theorem 3 and Lemma 5 .

As to the classes (VI)-(IX), since the signs of the orders of these classes are symmetric with respect to (IV), (III), (II) and (I), respectively, they can realize 288, 240, 160 and $104 \mathrm{LSBF}$, respectively.

The proof of Lemma 6 is thus completed.

From Lemma 6, we immediately obtain the following realization theorem for LSBF:

Theorem 4. There are only 1882 linearly separable Boolean functions or Boolean genes of four variables, i.e. 1882 Boolean functions can be realized by a standard uncoupled $C N N$ of four input variables.

We will list the binary decoding tapes, decimal codes and genes of output patterns of classes (I) and (IX) among these 1882 LSBF in the next section, leaving the rest to a separate supplement [Chen \& Chen, 2004].

\section{Genes, Binary Decoding Tapes and Decimal Codes of LSBF}

Based on the analysis and results given in the preceding sections, we are now able to realize 
exactly 1882 LSBF via the CNN (7), and calculate the corresponding genes, binary decoding tapes and decimal codes. The procedure is as follows.

Step 1. Take three different real numbers, $w_{0}$, $w_{1}$ and $w_{2}$, and let $w_{3}=-w_{0}+w_{1}+w_{2}$. After that, take another real number $w_{4}$ such that $w_{4} \neq$ $w_{i}(i=0,1,2,3)$, and let $w_{5}=-w_{0}+w_{1}+w_{4}$, $w_{6}=-w_{0}+w_{2}+w_{4}, w_{7}=-w_{0}+w_{3}+w_{4}$. Then, the eight numbers $w_{k}(k=0,1,2, \ldots, 7)$ form one of the 96 orders in the nine classes shown in Lemma 4:

$$
w_{i_{0}}<w_{i_{1}}<w_{i_{2}}<w_{i_{3}}<w_{i_{4}}<w_{i_{5}}<w_{i_{6}}<w_{i_{7}} .
$$

Step 2. Divide $(-\infty,+\infty)$ into nine open intervals by $w_{i_{j}} / 2(j=0,1,2, \ldots, 7)$, as follows:

$$
\begin{gathered}
I_{0}=\left(-\infty, \frac{w_{i_{0}}}{2}\right), \quad I_{1}=\left(\frac{w_{i_{0}}}{2}, \frac{w_{i_{1}}}{2}\right), \\
I_{2}=\left(\frac{w_{i_{1}}}{2}, \frac{w_{i_{2}}}{2}\right), \quad I_{3}=\left(\frac{w_{i_{2}}}{2}, \frac{w_{i_{3}}}{2}\right), \\
I_{4}=\left(\frac{w_{i_{3}}}{2}, \frac{w_{i_{4}}}{2}\right), \quad I_{5}=\left(\frac{w_{i_{4}}}{2}, \frac{w_{i_{5}}}{2}\right), \\
I_{6}=\left(\frac{w_{i_{5}}}{2}, \frac{w_{i_{6}}}{2}\right), \quad I_{7}=\left(\frac{w_{i_{6}}}{2}, \frac{w_{i_{7}}}{2}\right), \\
I_{8}=\left(\frac{w_{i_{7}}}{2},+\infty\right) .
\end{gathered}
$$

Step 3. For every interval $I_{j}(j=0,1,2, \ldots, 7)$, take a number $z_{j} \in I_{j}$ and calculate $b_{1}, b_{2}, b_{3}$ and $b_{4}$ by using $w_{0}, w_{1}, w_{2}, w_{4}$ and $z_{j}$ in the formula (19):

$$
\left(\begin{array}{l}
b_{1} \\
b_{2} \\
b_{3} \\
b_{4}
\end{array}\right)=A^{-1}\left(\begin{array}{l}
w_{0}-z_{j} \\
w_{1}-z_{j} \\
w_{2}-z_{j} \\
w_{4}-z_{j}
\end{array}\right) .
$$

Then, let

$$
\begin{aligned}
w_{8}=2 z_{j}-w_{7}, & w_{9}=2 z_{j}-w_{6}, \\
w_{10}=2 z_{j}-w_{5}, & w_{11}=2 z_{j}-w_{4}, \\
w_{12}=2 z_{j}-w_{3}, & w_{13}=2 z_{j}-w_{2}, \\
w_{14}=2 z_{j}-w_{1}, & w_{15}=2 z_{j}-w_{0} .
\end{aligned}
$$

At last, calculate the self-feedback coefficient $a$ :

$$
a=1+\frac{1}{2} \min \left\{\mid w_{k} \| k=0,1,2, \ldots, 15\right\} .
$$

Thus, the six numbers, $z_{j}, b_{1}, b_{2}, b_{3}, b_{4}$ and $a$, constitute a gene of CNN (7):

\begin{tabular}{|l|l|l|l|l|l|}
\hline$z_{j}$ & $b_{1}$ & $b_{2}$ & $b_{3}$ & $b_{4}$ & $a$ \\
\hline
\end{tabular}

Step 4. Calculate the output pattern of the corresponding gene in Step 3:

$$
\begin{aligned}
\left(v_{0}, v_{1}, v_{2}, \ldots, v_{15}\right)= & \left(\operatorname{sgn}\left(w_{0}\right), \operatorname{sgn}\left(w_{1}\right),\right. \\
& \left.\operatorname{sgn}\left(w_{2}\right), \ldots, \operatorname{sgn}\left(w_{15}\right)\right)
\end{aligned}
$$

and its binary decoding tapes $\bar{v}_{0} \bar{v}_{1} \bar{v}_{2} \cdots \bar{v}_{15}$ and decimal code

$$
p=\bar{v}_{0} 2^{14}+\bar{v}_{1} 2^{13}+\cdots+\bar{v}_{14} 2+\bar{v}_{15}
$$

where $\bar{v}_{k}=1$ if $v_{k}=1, \bar{v}_{k}=0$ if $v_{k}=-1$.

Remark 5. (1) It is easy to see that we can obtain $96 \times 9 \times 9=7776$ CNN genes from Steps 1 to 4, based on Theorem 3, but there are only 1882 output pattern of the CNN (7) according to Lemma 5 and Theorem 4. In other words, different genes can connect to the same output pattern. In such a situation, we only take a gene as the representative of the output pattern in the gene bank of the CNN (7).

(2) To be more precise, in general, eight numbers $w_{i}(i=0,1,2,3,4,5,6,7)$ are taken as even numbers, such that $a=1+(1 / 2) \min \left\{\mid w_{k} \| k=\right.$ $0,1,2, \ldots, 15\}=2$, and let $z_{j}$ be the center value of the interval $I_{j}(j \neq 0,8)$.

(3) The gene (or template) design method described above in this paper may be referred to as a threshold bifurcation method, which is different from the nine design tools provided in [Chua et al., 2002]. Obviously, the method presented here is more mathematically rigorous.

It would be desirable to list all the genes, binary decoding tapes, and decimal codes of the 1882 $\mathrm{LSBF}$ that can be realized via CNN. However, since the list is too long, only two classes (I) and (IX) will be shown in Tables 4 and 5, respectively, for demonstration. The rest will be supplied elsewhere [Chen \& Chen, 2004]. 
Table 4. Genes, binary decoding tapes, and decimal codes of LSBF of class (I).

\begin{tabular}{|c|c|c|c|c|c|c|c|c|c|}
\hline No. & Decimal Code & Gene: $\mathrm{z}$ & $b_{1}$ & $b$ & & $b_{3}$ & $b_{4}$ & $\mathrm{a}$ & Binary Decoding Tape \\
\hline 1 & 0 & -16 & 0 & 8 & 4 & 2 & 2 & & 0000000000000000 \\
\hline 2 & 1 & -14 & 2 & 8 & 4 & 2 & 2 & & 0000000000000001 \\
\hline 3 & 2 & -14 & 2 & 8 & 4 & -2 & 2 & & 0000000000000010 \\
\hline 4 & 3 & \begin{tabular}{|l}
-12 \\
\end{tabular} & 2 & 8 & 4 & 2 & 2 & & 0000000000000011 \\
\hline 5 & 4 & \begin{tabular}{|l|}
-14 \\
\end{tabular} & 2 & 8 & -2 & 4 & 2 & & 0000000000000100 \\
\hline 6 & 5 & -12 & 4 & 8 & 2 & 4 & 2 & & 0000000000000101 \\
\hline 7 & 7 & -10 & 6 & 8 & 4 & 2 & 2 & & 0000000000000111 \\
\hline 8 & 8 & -14 & 2 & 8 & -2 & $\overline{-4}$ & 2 & & 0000000000001000 \\
\hline 9 & 10 & -12 & 4 & 8 & 2 & -4 & 2 & & 0000000000001010 \\
\hline 10 & 11 & -10 & 6 & 8 & 4 & -2 & 2 & & 0000000000001011 \\
\hline 11 & 12 & \begin{tabular}{|l|}
-12 \\
\end{tabular} & 4 & 8 & -4 & 2 & 2 & & 0000000000001100 \\
\hline 12 & 13 & -10 & 6 & 8 & -2 & 4 & 2 & & 0000000000001101 \\
\hline 13 & 14 & -10 & 6 & 8 & -2 & -4 & 2 & & 0000000000001110 \\
\hline 14 & 15 & -8 & 8 & 8 & 4 & 2 & 2 & & 0000000000001111 \\
\hline 15 & 16 & -14 & 2 & -2 & 8 & 2 & 2 & & 0000000000010000 \\
\hline 16 & 17 & \begin{tabular}{|l|}
-12 \\
\end{tabular} & 4 & 2 & 8 & 2 & 2 & & 0000000000010001 \\
\hline 17 & 19 & -12 & 8 & 6 & 8 & 4 & 2 & & 0000000000010011 \\
\hline 18 & 21 & -12 & 8 & 6 & 4 & 8 & 2 & & 0000000000010101 \\
\hline 19 & 23 & -12 & 12 & 10 & 8 & 4 & 2 & & 0000000000010111 \\
\hline 20 & 31 & \begin{tabular}{|l}
-6 \\
\end{tabular} & 10 & 8 & 4 & 2 & 2 & & 0000000000011111 \\
\hline 21 & 32 & \begin{tabular}{|l|}
-14 \\
\end{tabular} & 2 & -2 & 8 & 4 & 2 & & 0000000000100000 \\
\hline 22 & 34 & -12 & 4 & 2 & 8 & -4 & 2 & & 0000000000100010 \\
\hline 23 & 35 & -12 & 8 & 6 & 8 & -4 & 2 & & 0000000000100011 \\
\hline 24 & 42 & \begin{tabular}{|l|}
-12 \\
\end{tabular} & 4 & 6 & 4 & -4 & 2 & & 0000000000101010 \\
\hline 25 & 43 & -12 & 12 & 10 & 8 & -4 & 2 & & 0000000000101011 \\
\hline 26 & 47 & -6 & 10 & 8 & 4 & -2 & 2 & & 0000000000101111 \\
\hline 27 & 48 & -14 & 6 & -6 & 8 & 2 & 2 & & 0000000000110000 \\
\hline 28 & 49 & -10 & 6 & -2 & 8 & 2 & 2 & & 0000000000110001 \\
\hline 29 & 50 & \begin{tabular}{|l|}
-10 \\
\end{tabular} & 6 & -2 & 8 & 4 & 2 & & 0000000000110010 \\
\hline 30 & 51 & -8 & 8 & 2 & 8 & 2 & 2 & & 0000000000110011 \\
\hline 31 & 55 & -8 & 12 & 6 & 8 & 4 & 2 & & 0000000000110111 \\
\hline 32 & 59 & -8 & 12 & 6 & 8 & -4 & 2 & & 0000000000111011 \\
\hline 33 & 63 & \begin{tabular}{|l|}
-4 \\
\end{tabular} & 12 & 8 & 4 & 2 & 2 & & 0000000000111111 \\
\hline 34 & 64 & -14 & 2 & -2 & -4 & 4 & 2 & & 0000000001000000 \\
\hline 35 & 68 & -12 & 4 & 2 & -4 & 8 & 2 & & 0000000001000100 \\
\hline
\end{tabular}


Table 4. (Continued)

\begin{tabular}{|c|c|c|c|c|c|c|c|c|c|}
\hline No. & Decimal Code & Gene: $\mathrm{z}$ & $b_{1}$ & $b_{2}$ & $\mathrm{~S}_{2}$ & $b_{3}$ & $b_{4}$ & $\mathrm{a}$ & Binary Decoding Tape \\
\hline 36 & 69 & -12 & 8 & 6 & -4 & 8 & 2 & & 0000000001000101 \\
\hline 37 & 76 & -12 & 8 & 6 & -8 & 4 & 2 & & 0000000001001100 \\
\hline 38 & 77 & -12 & 12 & 10 & $\mid-4$ & -4 & 2 & & 0000000001001101 \\
\hline 39 & 79 & -6 & 10 & 8 & -2 & 4 & 2 & & 0000000001001111 \\
\hline 40 & 80 & -14 & 6 & -6 & 4 & 8 & 2 & & 0000000001010000 \\
\hline 41 & 81 & \begin{tabular}{|l|}
-10 \\
\end{tabular} & 6 & -2 & 4 & 8 & 2 & & 0000000001010001 \\
\hline 42 & 84 & -10 & 6 & -2 & -4 & 4 & 2 & & 0000000001010100 \\
\hline 43 & 85 & -8 & 8 & 2 & 4 & 8 & 2 & & 0000000001010101 \\
\hline 44 & 87 & -8 & 12 & 6 & 4 & 8 & 2 & & 0000000001010111 \\
\hline 45 & 93 & -8 & 12 & 6 & -4 & 8 & 2 & & 0000000001011101 \\
\hline 46 & 95 & -4 & 12 & 8 & 2 & 4 & 2 & & 0000000001011111 \\
\hline 47 & 112 & -14 & 10 & -10 & 0 & 8 & 2 & & 0000000001110000 \\
\hline 48 & 113 & -10 & 10 & -6 & 6 & 2 & 2 & & 0000000001110001 \\
\hline 49 & 115 & -6 & 10 & -2 & 8 & 2 & 2 & & 0000000001110011 \\
\hline 50 & 117 & -6 & 10 & -2 & 4 & 8 & 2 & & 0000000001110101 \\
\hline 51 & 119 & \begin{tabular}{|l|}
-4 \\
\end{tabular} & 12 & 2 & 8 & 2 & 2 & & 0000000001110111 \\
\hline 52 & 127 & \begin{tabular}{|l}
-2 \\
\end{tabular} & 14 & 8 & 4 & 2 & 2 & & 0000000001111111 \\
\hline 53 & 128 & -14 & 2 & -2 & -4 & -8 & 2 & & 0000000010000000 \\
\hline 54 & 136 & -10 & 6 & 2 & -4 & -8 & 2 & & 0000000010001000 \\
\hline 55 & 138 & -12 & 8 & 6 & -4 & -8 & 2 & & 0000000010001010 \\
\hline 56 & 140 & -12 & 8 & 6 & -8 & -4 & 2 & & 0000000010001100 \\
\hline 57 & 142 & -12 & 12 & 10 & -4 & -8 & 8 & & 0000000010001110 \\
\hline 58 & 143 & -6 & 10 & 8 & -2 & -4 & 2 & & 0000000010001111 \\
\hline 59 & 160 & \begin{tabular}{|l|}
-14 \\
\end{tabular} & 6 & -6 & 4 & 8 & 2 & & 0000000010100000 \\
\hline 60 & 162 & -10 & 6 & -2 & 4 & -8 & 2 & & 0000000010100010 \\
\hline 61 & 168 & -10 & 6 & -2 & -4 & \begin{tabular}{|l|l|} 
& -8 \\
\end{tabular} & 2 & & 0000000010101000 \\
\hline 62 & 170 & \begin{tabular}{|l|}
-8 \\
\end{tabular} & 8 & 2 & \begin{tabular}{l|l}
4 & \\
\end{tabular} & -8 & 2 & & 0000000010101010 \\
\hline 63 & 171 & -8 & 8 & 6 & 4 & -4 & 2 & & 0000000010101011 \\
\hline 64 & 174 & -8 & 12 & 6 & -4 & -8 & 2 & & 0000000010101110 \\
\hline 65 & 175 & -4 & 12 & 8 & 2 & -4 & 2 & & 0000000010101111 \\
\hline 66 & 176 & -14 & 10 & -10 & 8 & \begin{tabular}{l|l}
8 & -4 \\
\end{tabular} & 4 & & 0000000010110000 \\
\hline 67 & 178 & -10 & 10 & -6 & 8 & -4 & 2 & & 0000000010110010 \\
\hline 68 & 179 & -6 & 10 & -2 & 8 & 4 & 2 & & 0000000010110011 \\
\hline 69 & 186 & -6 & 10 & -2 & 4 & -8 & 2 & & 0000000010111010 \\
\hline 70 & 187 & \begin{tabular}{|l|}
-4 \\
\end{tabular} & 12 & 2 & 8 & -4 & 2 & & 0000000010111011 \\
\hline 71 & 191 & -2 & 14 & 8 & 4 & -2 & 2 & & 0000000010111111 \\
\hline
\end{tabular}


Table 4. (Continued)

\begin{tabular}{|c|c|c|c|c|c|c|c|c|}
\hline No. & Decimal Code & Gene: 2 & $\mathrm{z}$ & $b_{2}$ & $b_{3}$ & $b_{4}$ & $\mathrm{a}$ & Binary Decoding Tape \\
\hline 72 & 192 & -14 & 6 & -6 & -8 & 4 & 2 & 0000000011000000 \\
\hline 73 & 196 & -10 & 6 & -2 & -8 & 4 & 2 & 0000000011000100 \\
\hline 74 & 200 & -10 & 6 & -2 & -8 & -4 & 2 & 0000000011001000 \\
\hline 75 & 204 & -8 & 8 & 2 & -8 & 4 & 2 & 0000000011001100 \\
\hline 76 & 205 & -8 & 12 & 6 & -8 & 4 & 2 & 0000000011001101 \\
\hline 77 & 206 & -8 & 12 & 6 & -8 & -4 & 2 & 0000000011001110 \\
\hline 78 & 207 & -4 & 12 & 8 & -4 & 2 & 2 & 0000000011001111 \\
\hline 79 & 208 & -14 & 10 & -10 & -4 & 8 & 2 & 0000000011010000 \\
\hline 80 & 212 & \begin{tabular}{|l|}
-10 \\
\end{tabular} & 10 & -6 & -4 & 8 & 2 & 0000000011010100 \\
\hline 81 & 213 & -6 & 10 & -2 & -4 & 8 & 2 & 0000000011010101 \\
\hline 82 & 220 & -6 & 10 & -2 & -8 & 4 & 2 & 0000000011011100 \\
\hline 83 & 221 & \begin{tabular}{|l|}
-4 \\
\end{tabular} & 12 & 2 & -4 & 8 & 2 & 0000000011011101 \\
\hline 84 & 223 & -2 & 14 & 8 & -2 & 4 & 2 & 0000000011011111 \\
\hline 85 & 224 & \begin{tabular}{l|l}
-14 & \\
\end{tabular} & 10 & -10 & -4 & -8 & 2 & 0000000011100000 \\
\hline 86 & 232 & -10 & 10 & -6 & -4 & -8 & 2 & 0000000011101000 \\
\hline 87 & 234 & -6 & 10 & -2 & -4 & -8 & 2 & 0000000011101010 \\
\hline 88 & 236 & -6 & 10 & -2 & -8 & -4 & 2 & 0000000011101100 \\
\hline 89 & 238 & \begin{tabular}{|l|}
-6 \\
\end{tabular} & 10 & 2 & -4 & -8 & 2 & 0000000011101110 \\
\hline 90 & 239 & \begin{tabular}{|l|}
-2 \\
\end{tabular} & 14 & 8 & -2 & -4 & 2 & 0000000011101111 \\
\hline 91 & 240 & -8 & 8 & -8 & 4 & \begin{tabular}{l|l}
2 & \\
\end{tabular} & 2 & 0000000011110000 \\
\hline 92 & 241 & \begin{tabular}{|l|}
-10 \\
\end{tabular} & 14 & -10 & \begin{tabular}{|l|l|}
0 & 8 \\
\end{tabular} & 2 & 2 & 0000000011110001 \\
\hline 93 & 242 & -10 & 14 & -10 & 8 & -4 & 2 & 0000000011110010 \\
\hline 94 & 243 & \begin{tabular}{|l|}
-6 \\
\end{tabular} & 14 & -6 & -8 & \begin{tabular}{|l|l|}
2 \\
\end{tabular} & 2 & 0000000011110011 \\
\hline 95 & 244 & -10 & 14 & -10 & -4 & 8 & \begin{tabular}{l|l}
2 \\
\end{tabular} & 0000000011110100 \\
\hline 96 & 245 & -6 & 14 & -6 & 4 & & 2 & 0000000011110101 \\
\hline 97 & 247 & \begin{tabular}{|l|}
-2 \\
\end{tabular} & 14 & -2 & 8 & & 2 & 0000000011110111 \\
\hline 98 & 248 & -10 & 14 & -10 & -4 & -8 & 2 & 0000000011111000 \\
\hline 99 & 250 & -6 & 14 & -6 & 4 & & 2 & 0000000011111010 \\
\hline 100 & 251 & \begin{tabular}{|l}
-2 \\
\end{tabular} & 14 & -2 & 8 & & 2 & 0000000011111011 \\
\hline 101 & 252 & -6 & 14 & -6 & -8 & 4 & 2 & 0000000011111100 \\
\hline 102 & 253 & -2 & 14 & -2 & -4 & 8 & 2 & 0000000011111101 \\
\hline 103 & 254 & -2 & 14 & -2 & -4 & -8 & 2 & 0000000011111110 \\
\hline 104 & 255 & 0 & 16 & 8 & 4 & 2 & 2 & 0000000011111111 \\
\hline
\end{tabular}


Table 5. Genes, binary decoding tapes, and decimal codes of LSBF of class (IX).

\begin{tabular}{|c|c|c|c|c|c|c|c|c|c|}
\hline No. & Decimal Code & Gene: [ & $\mathrm{z}$ & $b_{2}$ & 2 & $b_{3}$ & $b_{4}$ & $\mathrm{a}$ & Binary Decoding Tape \\
\hline 1779 & 65280 & 0 & -16 & 8 & 4 & 2 & 2 & & 1111111100000000 \\
\hline 1780 & 65281 & 2 & -14 & 8 & 4 & 2 & 2 & & 1111111100000001 \\
\hline 1781 & 65282 & 2 & -14 & 8 & 4 & -2 & 2 & & 1111111100000010 \\
\hline 1782 & 65283 & 2 & -12 & 8 & 4 & 2 & 2 & & 1111111100000011 \\
\hline 1783 & 65284 & 2 & -14 & 8 & -2 & 4 & 2 & & 1111111100000100 \\
\hline 1784 & 65285 & 4 & -12 & 8 & 2 & 4 & 2 & & 1111111100000101 \\
\hline 1785 & 65287 & 6 & -10 & 8 & 4 & 2 & 2 & & 1111111100000111 \\
\hline 1786 & 65288 & 2 & -14 & 8 & -2 & -4 & 2 & & 1111111100001000 \\
\hline 1787 & 65290 & 4 & -12 & 8 & 2 & -4 & 2 & & 1111111100001010 \\
\hline 1788 & 65291 & 6 & -10 & 8 & 4 & -2 & 2 & & 1111111100001011 \\
\hline 1789 & 65292 & 4 & -12 & 8 & -4 & 2 & 2 & & 1111111100001100 \\
\hline 1790 & 65293 & 6 & -10 & 8 & -2 & 4 & 2 & & 1111111100001101 \\
\hline 1791 & 65294 & 6 & -10 & 8 & -2 & -4 & 2 & & 1111111100001110 \\
\hline 1792 & 65295 & 8 & -8 & 8 & 4 & 2 & 2 & & 1111111100001111 \\
\hline 1793 & 65296 & 2 & -14 & -2 & 8 & 2 & 2 & & 1111111100010000 \\
\hline 1794 & 65297 & 4 & -12 & 2 & 8 & 2 & 2 & & 1111111100010001 \\
\hline 1795 & 65299 & 8 & -12 & 6 & 8 & 4 & 2 & & 1111111100010011 \\
\hline 1796 & 65301 & 8 & -12 & 6 & 4 & 8 & 2 & & 1111111100010101 \\
\hline 1797 & 65303 & 12 & -12 & 10 & 8 & 4 & 2 & & 1111111100010111 \\
\hline 1798 & 65311 & 10 & -6 & 8 & 4 & 2 & 2 & & 1111111100011111 \\
\hline 1799 & 65312 & 2 & -14 & -2 & 8 & 4 & 2 & & 1111111100100000 \\
\hline 1800 & 65314 & 4 & -12 & 2 & 8 & -4 & 2 & & 1111111100100010 \\
\hline 1801 & 65315 & 8 & -12 & 6 & 8 & -4 & 2 & & 1111111100100011 \\
\hline 1802 & 65322 & 4 & -12 & 6 & 4 & -4 & 2 & & 1111111100101010 \\
\hline 1803 & 65323 & 12 & -12 & 10 & 8 & -4 & 2 & & 1111111100101011 \\
\hline 1804 & 65327 & 10 & -6 & 8 & 4 & -2 & 2 & & 1111111100101111 \\
\hline 1805 & 65328 & 6 & -14 & -6 & 8 & 2 & 2 & & 1111111100110000 \\
\hline 1806 & 65329 & 6 & -10 & -2 & 8 & 2 & 2 & & 1111111100110001 \\
\hline 1807 & 65330 & 6 & -10 & -2 & 8 & 4 & 2 & & 1111111100110010 \\
\hline 1808 & 65331 & 8 & -8 & 2 & 8 & 2 & 2 & & 1111111100110011 \\
\hline 1809 & 65335 & 12 & -8 & 6 & 8 & 4 & 2 & & 1111111100110111 \\
\hline 1810 & 65339 & 12 & -8 & 6 & 8 & -4 & 2 & & 1111111100111011 \\
\hline 1811 & 65343 & 12 & -4 & 8 & 4 & 2 & 2 & & 1111111100111111 \\
\hline 1812 & 65344 & 2 & -14 & -2 & -4 & 8 & 2 & & 1111111101000000 \\
\hline 1813 & 65348 & 4 & -12 & 2 & -4 & 8 & 2 & & 1111111101000100 \\
\hline 1814 & 65349 & 8 & -12 & 6 & -4 & 8 & 2 & & 1111111101000101 \\
\hline
\end{tabular}


Table 5. (Continued)

\begin{tabular}{|c|c|c|c|c|c|c|c|c|c|}
\hline No. & Decimal Code & Gene: & \begin{tabular}{|l|}
$\mathrm{z}$ \\
\end{tabular} & $b_{1}$ & $b_{2}$ & $b_{3}$ & & $b_{4}$ & Binary Decoding Tape \\
\hline 1815 & 65356 & 8 & -12 & 6 & - & -8 & 4 & 2 & 1111111101001100 \\
\hline 1816 & 65357 & 12 & -12 & 10 & 0 & -4 & 8 & 2 & 1111111101001101 \\
\hline 1817 & 65359 & 10 & \begin{tabular}{l|l}
0 & -6
\end{tabular} & 8 & - & -2 & 4 & 2 & 1111111101001111 \\
\hline 1818 & 65360 & 6 & -14 & -6 & & 4 & 8 & 2 & 1111111101010000 \\
\hline 1819 & 65361 & 6 & -10 & $\overline{-2}$ & & 4 & 8 & 2 & 1111111101010001 \\
\hline 1820 & 65364 & 6 & -10 & -2 & & -4 & 8 & 2 & 1111111101010100 \\
\hline 1821 & 65365 & $\varepsilon$ & -8 & 2 & 4 & 4 & 8 & 2 & 1111111101010101 \\
\hline 1822 & 65367 & 1 & \begin{tabular}{l|l|}
2 & $-\varepsilon$ \\
\end{tabular} & -8 & 6 & 4 & 8 & 2 & 1111111101010111 \\
\hline 1823 & 65373 & 12 & -8 & 6 & - & -4 & 8 & 2 & 1111111101011101 \\
\hline 1824 & 65375 & 1 & \begin{tabular}{l|l|}
2 & -4 \\
\end{tabular} & -4 & 8 & 2 & 4 & 2 & 1111111101011111 \\
\hline 1825 & 65392 & 10 & -14 & -1 & 10 & 8 & 2 & 2 & 1111111101110000 \\
\hline 1826 & 65393 & 10 & -10 & -1 & -6 & 8 & 2 & 2 & 1111111101110001 \\
\hline 1827 & 65395 & 10 & -6 & -2 & & 8 & 2 & 2 & 1111111101110011 \\
\hline 1828 & 65397 & 10 & -6 & -2 & & 4 & 8 & 2 & 1111111101110101 \\
\hline 1829 & 65399 & 1 & \begin{tabular}{|l|l|}
2 & -4 \\
\end{tabular} & -4 & 2 & 8 & 2 & 2 & 1111111101110111 \\
\hline 1830 & 65407 & \begin{tabular}{|l}
1 \\
\end{tabular} & \begin{tabular}{l|l}
4 & -2 \\
\end{tabular} & -2 & 8 & 4 & 2 & 2 & 1111111101111111 \\
\hline 1831 & 65408 & 2 & -14 & -2 & - & -4 & -8 & 2 & 1111111110000000 \\
\hline 1832 & 65416 & 6 & -10 & 2 & -4 & & -8 & 2 & 1111111110001000 \\
\hline 1833 & 65418 & 8 & -12 & 6 & -4 & & -8 & 2 & 1111111110001010 \\
\hline 1834 & 65420 & 8 & -12 & 6 & $-\varepsilon$ & & -4 & 2 & 1111111110001100 \\
\hline 1835 & 65422 & 12 & -12 & 10 & - & -4 & -8 & 2 & 1111111110001110 \\
\hline 1836 & 65423 & 10 & \begin{tabular}{|l|l|} 
& -6 \\
\end{tabular} & 8 & -2 & & -4 & 2 & 1111111110001111 \\
\hline 1837 & 65440 & 6 & -14 & -6 & & 4 & 8 & 2 & 1111111110100000 \\
\hline 1838 & 65442 & 6 & -10 & -2 & 4 & 4 & -8 & 2 & 1111111110100010 \\
\hline 1839 & 65448 & 6 & -10 & -2 & - & -4 & -8 & 2 & 1111111110101000 \\
\hline 1840 & 65450 & 8 & \begin{tabular}{l|l}
3 & -8 \\
\end{tabular} & 2 & 4 & L & -8 & 2 & 1111111110101010 \\
\hline 1841 & 65451 & 8 & \begin{tabular}{|l|l|}
3 & -8 \\
\end{tabular} & 6 & 4 & - & -4 & 2 & 1111111110101011 \\
\hline 1842 & 65454 & 12 & \begin{tabular}{l|l}
-8 \\
\end{tabular} & 6 & -4 & & -8 & 2 & 1111111110101110 \\
\hline 1843 & 65455 & 12 & -4 & 8 & 2 & 21 & -4 & 2 & 1111111110101111 \\
\hline 1844 & 65456 & 10 & -14 & -1 & & 8 & -4 & 2 & 1111111110110000 \\
\hline 1845 & 65458 & 10 & -10 & -6 & & 8 & -4 & 2 & 1111111110110010 \\
\hline 1846 & 65459 & 10 & -6 & -2 & & 8 & 4 & 2 & 1111111110110011 \\
\hline 1847 & 65466 & 10 & -6 & -2 & 4 & 4 & -8 & 2 & 1111111110111010 \\
\hline 1848 & 65467 & 12 & -4 & 2 & 8 & 3 & -4 & 2 & 1111111110111011 \\
\hline 1849 & 65471 & 14 & -2 & 8 & 4 & 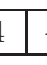 & -2 & 2 & 1111111110111111 \\
\hline 1850 & 65472 & 6 & -14 & -6 & & -8 & 4 & 2 & 1111111111000000 \\
\hline
\end{tabular}


Table 5. (Continued)

\begin{tabular}{|c|c|c|c|c|c|c|c|c|c|c|}
\hline No. & Decimal Code & Gene: & $\mathrm{z}$ & $b_{1}$ & $b_{2}$ & $b_{3}$ & & $b_{4}$ & $\mathrm{a}$ & Binary Decoding Tape \\
\hline 1851 & 65476 & 6 & -10 & & -2 & -8 & 4 & 2 & & 1111111111000100 \\
\hline 1852 & 65480 & 6 & -10 & & -2 & -8 & -4 & 2 & 2 & 1111111111001000 \\
\hline 1853 & 65484 & 8 & -8 & & 2 & -8 & 4 & 2 & & 1111111111001100 \\
\hline 1854 & 65485 & 12 & \begin{tabular}{l|l}
2 & -8 \\
\end{tabular} & & 6 & -8 & 4 & 2 & & 1111111111001101 \\
\hline 1855 & 65486 & 12 & -8 & & 6 & -8 & -4 & 2 & & 1111111111001110 \\
\hline 1856 & 65487 & \begin{tabular}{|l|}
12 \\
\end{tabular} & \begin{tabular}{l|l}
2 & -4 \\
\end{tabular} & & 8 & -4 & 2 & 2 & & 1111111111001111 \\
\hline 1857 & 65488 & 10 & -14 & & -10 & -4 & & 8 & 2 & 1111111111010000 \\
\hline 1858 & 65492 & 10 & -10 & & -6 & -4 & 8 & 2 & & 1111111111010100 \\
\hline 1859 & 65493 & 10 & -6 & & -2 & -4 & 8 & 2 & & 1111111111010101 \\
\hline 1860 & 65500 & 10 & -6 & & -2 & -8 & 4 & 2 & & 1111111111011100 \\
\hline 1861 & 65501 & 12 & \begin{tabular}{l|l}
2 & -4 \\
\end{tabular} & & 2 & -4 & 8 & 2 & & 1111111111011101 \\
\hline 1862 & 65503 & 14 & -2 & & 8 & -2 & 4 & 2 & & 1111111111011111 \\
\hline 1863 & 65504 & 10 & -14 & & 10 & -4 & - & -8 & 2 & 1111111111100000 \\
\hline 1864 & 65512 & 10 & -10 & & -6 & -4 & -8 & 8 & 2 & 1111111111101000 \\
\hline 1865 & 65514 & 10 & -6 & & -2 & -4 & -8 & 2 & & 1111111111101010 \\
\hline 1866 & 65516 & 10 & -6 & & 2 & -8 & -4 & 2 & 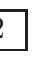 & 1111111111101100 \\
\hline 1867 & 65518 & 10 & -6 & & 2 & -4 & -8 & 2 & & 1111111111101110 \\
\hline 1868 & 65519 & 14 & -2 & & 8 & -2 & -4 & 2 & & 1111111111101111 \\
\hline 1869 & 65520 & 8 & -8 & & -8 & 4 & 2 & 2 & & 1111111111110000 \\
\hline 1870 & 65521 & 14 & -10 & & -10 & 8 & 2 & 2 & & 1111111111110001 \\
\hline 1871 & 65522 & 14 & -10 & & -10 & 8 & -4 & 2 & 2 & 1111111111110010 \\
\hline 1872 & 65523 & 14 & -6 & & -6 & -8 & 2 & 2 & & 1111111111110011 \\
\hline 1873 & 65524 & 14 & -10 & & -10 & -4 & 8 & 8 & 2 & 1111111111110100 \\
\hline 1874 & 65525 & 14 & \begin{tabular}{l|l} 
& -6 \\
\end{tabular} & & -6 & 4 & 8 & 2 & & 1111111111110101 \\
\hline 1875 & 65527 & 14 & \begin{tabular}{l|l} 
& -2 \\
\end{tabular} & & -2 & 8 & 2 & 2 & & 1111111111110111 \\
\hline 1876 & 65528 & \begin{tabular}{l|l}
14 & \\
\end{tabular} & -10 & & 10 & -4 & & 8 & 2 & 1111111111111000 \\
\hline 1877 & 65530 & 14 & -6 & & -6 & 4 & 8 & 2 & & 1111111111111010 \\
\hline 1878 & 65531 & \begin{tabular}{|l|}
14 \\
\end{tabular} & \begin{tabular}{l|l} 
& -2 \\
\end{tabular} & & -2 & 8 & 4 & 2 & & 1111111111111011 \\
\hline 1879 & 65532 & 14 & -6 & & -6 & -8 & 4 & 2 & & 1111111111111100 \\
\hline 1880 & 65533 & 14 & -2 & & -2 & -4 & 8 & 2 & & 1111111111111101 \\
\hline 1881 & 65534 & 14 & -2 & & 2 & -4 & -8 & 2 & & 1111111111111110 \\
\hline 1882 & 65535 & & 16 & 0 & 8 & 4 & 2 & 2 & & 1111111111111111 \\
\hline
\end{tabular}




\section{Conclusions}

In this paper, we have characterized some essential properties of an uncoupled CNN of four input variables. We have not only rigorously proved that the uncoupled CNN can realize exactly 1882 linearly separable Boolean functions (LSBF) or linearly separable Boolean genes (LSBG), but have also developed an effective method for generating all these CNN genes. In particular, we have established the CNN gene bank of four input variables, which contains all LSBG.

It is well known that a single CNN gene is the most important element for constructing the CNN chromosome. The more the CNN genes, the shorter the length of the corresponding CNN chromosome, and the more convenient the corresponding implementation task. The game-of-life chromosome, for example, consists of only two LSBG and one logical AND gene, which is already very powerful.

The CNN considered in this paper is the simplest possible two-dimensional locally-connected network. It can be easily implemented in applications such as image processing, brain science and pattern recognition. It is also possible that some functions of input-output operations of the CNN with nine input variables can be replaced by that of the CNN with four input variables studied in this paper.

Future research along the same line includes the establishment of more general results about the standard CNN of $n$ input variables and the discovery of more CNN genes that can realize all LSBF of $n$ variables, especially for the case of $n=9$. Such a huge bank of CNN genes is needed to be built, with which the CNN technology will find more engineering and technological applications.

\section{References}

Berlekamp, E. R., Conway, J. H. \& Guy, H. K. [1982] Winning Ways for Your Mathematical Plays (Academic Press, NY).

Chen, F. Y. \& Chen, G. [2004] "A complete list of genes, binary decoding tapes, and decimal codes of the $1882 \mathrm{LSBF}$ that can be realized via a CNN of four input variables," available at http://www.ee.cityu.edu.hk/ gchen/pdf/list.pdf

Chua, L. O., Dessor, C. A. \& Kuh, E. A. [1985] Linear and Nonlinear Circuits (McGraw Hill, NY).

Chua, L. O. \& Yang, L. [1988a] "Cellular neural networks: Theory," IEEE Trans. Circuits Syst. 35, 12571272 .

Chua, L. O. \& Yang, L. [1988b] "Cellular neural networks: Application," IEEE Trans. Circuits Syst. 35, 1273-1290.

Chua, L. O. \& Roska, T. [1993] "The CNN paradigm," IEEE Trans. Circuit Syst.-I 40, 147-156.

Chua, L. O. [1997] "CNN: A vision of complexity," Int. J. Bifurcation and Chaos 7, 2219-2425.

Chua, L. O. [1999] "CNN: A paradigm for complexity," Visions of Nonlinear Science in the 21st Century, Chap. 13 (World Scientific, Singapore).

Chua, L. O. \& Roska, T. [2002] Cellular Neural Networks and Visual Computing, Foundations and Applications (Cambridge University Press, UK).

Dogaru, R. \& Chua, L. O. [1999] "Universal CNN cells," Int. J. Bifurcation and Chaos 9, 1-48.

Hanggi, M. \& Moschytz, G. S. [1999] "An exact and direct analytical method for the design of optimally robust CNN templates," IEEE Trans. Circuit Syst.-I 46, 304-311.

Julian, P., Dogaru, R. \& Chua, L. O. [2002] "A piecewiselinear simplicial coupling cell for CNN gray-level image processing," IEEE Trans. Circuit Syst.-I 49, 904-913.

Julian, P., Dogaru, R., Itoh, M. \& Chua, L. O. [2003] "Simplicial RTD-based cellular nonlinear networks," IEEE Trans. Circuit Syst.-I 50, 500-509.

Kozek, T., Roska, T. \& Chua, L. O. [1993] "Genetic algorithm for CNN template learning," IEEE Trans. Circuit Syst.-I 40, 392-402.

Roska, T. \& Chua, L. O. [1993] "The CNN universal machine: An analogic array computer," IEEE Trans. Circuit Syst.-II 40, 163-173.

Roska, T., Kek, L., Nemes, L. \& Zarandy, A. [1997] "CNN Software Library (Templates and Algorithms)," Version 7.0 (DNS-1-1997). Analogical and Neural Computing Laboratory, Computer Automation Institute, Hungarian Academy of Sciences, Budapest, Hungary.

Yang, T., Crounse, K. R. \& Chua, L. O. [2001] "Spherical cellular nonlinear networks," Int. J. Bifurcation and Chaos 11, 241-257. 